Pacific

Journal of

Mathematics

\title{
2-TORUS MANIFOLDS, COBORDISM AND SMALL COVERS
}

\section{ZHI LÜ}




\title{
2-TORUS MANIFOLDS, COBORDISM AND SMALL COVERS
}

\author{
ZHI LÜ
}

Dedicated to Professor Robert E. Stong, who left us recently.

\begin{abstract}
Define $\mathfrak{M}_{n}$ to be the set of equivariant, unoriented cobordism classes of $\boldsymbol{n}$ dimensional 2-torus manifolds, where any such manifold is smooth, closed and $\boldsymbol{n}$-dimensional, and has an effective smooth action of a rank $\boldsymbol{n}$ 2-torus group $\left(\mathbb{Z}_{2}\right)^{n}$. Then $\mathfrak{M}_{n}$ forms an abelian group with respect to disjoint union. For $n=3$, we determine the group structure of $\mathfrak{M}_{n}$ and show that each class of $\mathfrak{M}_{n}$ contains a small cover as its representative.
\end{abstract}

\section{Introduction}

An $n$-dimensional 2-torus manifold $M$ is a smooth closed manifold of dimension $n$ with an effective smooth action of a rank $n$ 2-torus group $\left(\mathbb{Z}_{2}\right)^{n}$. Since the action is effective, the fixed point set of the action is 0-dimensional (that is, it is formed by finitely many isolated points) if $M$ has a fixed point. In this paper, we shall study this class of geometrical objects from the viewpoint of cobordism.

We denote by $\mathfrak{M}_{n}$ the set of equivariant unoriented cobordism classes of all $n$-dimensional 2-torus manifolds. Then $\mathfrak{M}_{n}$ forms an abelian group with respect to disjoint union, and in particular $\mathfrak{M}_{n}$ also forms a vector space over $\mathbb{Z}_{2}$. The zero element of $\mathfrak{M}_{n}$ can be represented by a canonical 2-torus manifold, which is the $n$-dimensional standard sphere $S^{n}$ with the standard $\left(\mathbb{Z}_{2}\right)^{n}$-action defined by $\left(x_{0}, x_{1}, \ldots, x_{n}\right) \mapsto\left(x_{0}, g_{1} x_{1}, \ldots, g_{n} x_{n}\right)$; this action fixes two isolated points with same $\left(\mathbb{Z}_{2}\right)^{n}$-representation, where $\left(x_{0}, x_{1}, \ldots, x_{n}\right) \in S^{n}$ and $\left(g_{1}, \ldots, g_{n}\right) \in\left(\mathbb{Z}_{2}\right)^{n}$. When $n=1,2$, it is known from [Conner and Floyd 1964] that $\mathfrak{M}_{1}$ is trivial and $\mathfrak{M}_{2}$ is generated by the standard $\left(\mathbb{Z}_{2}\right)^{2}$-action on $\mathbb{R} P^{2}$.

Problem. Determine the group structure of $\mathfrak{M}_{n}$ when $n \geq 3$.

Davis and Januszkiewicz [1991] introduced and studied small covers, which are special kinds of 2-torus manifolds; each small cover is locally isomorphic to a faithful representation of $\left(\mathbb{Z}_{2}\right)^{n}$ on $\mathbb{R}^{n}$, and its orbit space is a simple convex polytope. This establishes a direct link between equivariant topology and combinatorics. A

MSC2000: primary 55N22, 57R85, 57S17; secondary 05C10, 57M60.

Keywords: 2-torus manifolds, cobordism, small cover.

Supported by NSFC, grant numbers 10371020 and 10671034 . 
typical example of an equivariant nonbounding small cover is real projective space $\mathbb{R} P^{n}$ with the standard action of $\left(\mathbb{Z}_{2}\right)^{n}$. Its orbit space is an $n$-simplex. Another example of a bounding small cover is a product of $n$ copies of a circle $S^{1}$ with reflection; its orbit space is an $n$-cube. Thus we see that for $n=2$ these two examples can be used as representatives of the two classes of $\mathfrak{M}_{2}$.

Conjecture. Each class of $\mathfrak{M}_{n}$ contains a small cover as its representative.

Bukhshtaber and Ray [1998] proved this conjecture in nonequivariant case. In Theorems 6.6 and 7.2, we settle the above problem and conjecture for $n=3$.

The paper is organized as follows. In Section 2, we introduce and study some properties of an equivariant cobordism invariant (that is, the prime tangent representation set $\mathcal{N}_{\beta}$ ) associated to 2-torus manifolds coming from the Stong homomorphism. In Section 3, we introduce the notion of an essential generator of $\mathfrak{M}_{n}$, and show that any element of $\mathfrak{M}_{n}$ is a linear combination of essential generators. In Section 4, we review [Davis and Januszkiewicz 1991] and give two kinds of 3-dimensional small covers that play a key role in the study of $\mathfrak{M}_{3}$. In Section 5, we introduce the moment graphs induced by 2 -torus manifolds. The group structure of $\mathfrak{M}_{3}$ is determined completely in Section 6, and the conjecture above is settled in the 3-dimensional case in Section 7.

\section{2. $G$-representations and Stong homomorphism}

Let $G=\left(\mathbb{Z}_{2}\right)^{n}$, and let $\operatorname{Hom}\left(G, \mathbb{Z}_{2}\right)$ be the set of all homomorphisms $\rho: G \rightarrow \mathbb{Z}_{2}$, which consists of $2^{n}$ distinct homomorphisms. Let $\rho_{0}$ denote the trivial element in $\operatorname{Hom}\left(G, \mathbb{Z}_{2}\right)$, that is, $\rho_{0}(g)=1$ for all $g \in G$. The irreducible real $G$-representations are all one-dimensional and correspond to all elements in $\operatorname{Hom}\left(G, \mathbb{Z}_{2}\right)$. Thus, every irreducible real representation of $G$ has the form $\lambda_{\rho}: G \times \mathbb{R} \rightarrow \mathbb{R}$ with $\lambda_{\rho}(g, x)=\rho(g) \cdot x$ for $\rho \in \operatorname{Hom}\left(G, \mathbb{Z}_{2}\right)$.

Given an element $\beta$ of $\mathfrak{M}_{n}$, let $(M, \phi)$ be a representative of $\beta$ such that $M$ has a fixed point. Taking an isolated point $p$ in $M^{G}$, the $G$-representation at $p$ can be written as

$$
\tau_{p} M=\bigoplus_{\rho \neq \rho_{0}} \lambda_{\rho}^{q_{\rho}}
$$

with $\sum_{\rho \neq \rho_{0}} q_{\rho}=n$. By the Borel theorem (see [Allday and Puppe 1993]) and the effectiveness of the action, if $q_{\rho} \neq 0$, then $q_{\rho}$ must be one. Thus, $\tau_{p} M$ is the direct sum of $n$ irreducible real $G$-representations (which are linearly independent). The collection $\mathcal{N}_{(M, \phi)}=\left\{\left[\tau_{p} M\right] \mid p \in M^{G}\right\}$ is called the tangent representation set of $(M, \phi)$, where $\left[\tau_{p} M\right]$ denotes the isomorphism class of $\tau_{p} M$.

Denote by $R_{n}(G)$ the vector space over $\mathbb{Z}_{2}$ generated by the representation classes of dimension $n$. Then $R_{*}(G)=\sum_{n \geq 0} R_{n}(G)$ is a graded commutative algebra over $\mathbb{Z}_{2}$ with unit. The multiplication in $R_{*}(G)$ is given by $\left[V_{1}\right] \cdot\left[V_{2}\right]=\left[V_{1} \oplus V_{2}\right]$. 
We can identify $R_{*}(G)$ with the graded polynomial algebra over $\mathbb{Z}_{2}$ generated by $\operatorname{Hom}\left(G, \mathbb{Z}_{2}\right)$, where the addition in $\operatorname{Hom}\left(G, \mathbb{Z}_{2}\right)$ is given by the tensor product of representations $(\rho+\mu)(g)=\rho(g) \cdot \mu(g)$ and the multiplication is given by the direct sum of representations. The homomorphisms $\rho_{i}:\left(g_{1}, \ldots, g_{n}\right) \mapsto g_{i}$ give a standard basis of $\operatorname{Hom}\left(G, \mathbb{Z}_{2}\right)$. Then $R_{*}(G)$ is isomorphic to the graded polynomial algebra $\mathbb{Z}_{2}\left[\rho_{1}, \ldots, \rho_{n}\right]$. Obviously, each $\left[\tau_{p} M\right]$ of $\mathcal{N}_{(M, \phi)}$ corresponds to a unique homogeneous monomial of degree $n$ in $\mathbb{Z}_{2}\left[\rho_{1}, \ldots, \rho_{n}\right]$ such that the $n$ factors of the monomial form a basis of $\operatorname{Hom}\left(G, \mathbb{Z}_{2}\right)$.

There is a natural homomorphism $\delta_{n}: \mathfrak{M}_{n} \rightarrow R_{n}(G)$ defined by

$$
\delta_{n}([M, \phi])=\sum_{p \in M^{G}}\left[\tau_{p} M\right] .
$$

Theorem 2.1 [Stong 1970]. $\delta_{n}$ is a monomorphism.

This implies that for each $\beta$ in $\mathfrak{M}_{n}$, there must be a representative $(M, \phi)$ of $\beta$ such that $\mathcal{N}_{(M, \phi)}$ is prime (that is, either all elements of $\mathcal{N}_{(M, \phi)}$ are distinct or $\mathcal{N}_{(M, \phi)}$ is empty), and $\mathcal{N}_{(M, \phi)}$ is uniquely determined by $\beta$. Define $\mathcal{N}_{\beta}:=\mathcal{N}_{(M, \phi)}$ and call it the prime tangent representation set of $\beta$.

Corollary 2.2. Let $\beta_{1}, \beta_{2} \in \mathfrak{M}_{n}$. Then $\beta_{1}=\beta_{2}$ if and only if $\mathcal{N}_{\beta_{1}}=\mathcal{N}_{\beta_{2}}$.

Remark 2.1. Since $\operatorname{Hom}\left(G, \mathbb{Z}_{2}\right)$ is isomorphic to $G$, each $\left[\tau_{p} M\right]$ of $\mathcal{N}_{(M, \phi)}$ actually corresponds a unique element (denoted by $\left[\Delta_{p}\right]$ ) in the quotient $\operatorname{GL}\left(n, \mathbb{Z}_{2}\right) / \mathbf{S}_{n}$, where $\mathbf{S}_{n}$ is the subgroup generated by all matrices of the form $E_{i j}$ (that is, the identity matrix $E$, but with columns $i$ and $j$ exchanged); $\mathbf{S}_{n}$ is isomorphic to the symmetric group of rank $n$. Thus, for any two $\sigma_{1}, \sigma_{2}$ in $\left[\Delta_{p}\right]$, there exists a matrix $\theta$ in $\mathbf{S}_{n}$ such that $\sigma_{1}=\sigma_{2} \theta$. This also means that there is a one-to-one correspondence between bases in $\left(\mathbb{Z}_{2}\right)^{n}$ and bases in $\operatorname{GL}\left(n, \mathbb{Z}_{2}\right) / \mathbf{S}_{n}$. Here we call $\left[\Delta_{p}\right]$ the tangent matrix at $p$. With this understanding, we often regard each element $\left[\tau_{p} M\right]$ of $\mathcal{N}_{(M, \phi)}$ as being $\left[\Delta_{p}\right]$. Note that $\left|\mathrm{GL}\left(n, \mathbb{Z}_{2}\right)\right|=2^{n(n-1) / 2} \prod_{i=1}^{n}\left(2^{i}-1\right)$; see [Alperin and Bell 1995].

Proposition 2.3. Let $\beta$ be a nonzero element of $\mathfrak{M}_{n}$. Then

$$
n+1 \leq\left|\mathcal{N}_{\beta}\right| \leq \frac{2^{n(n-1) / 2} \prod_{i=1}^{n}\left(2^{i}-1\right)}{n !} .
$$

Further, such upper and lower bounds are the best possible.

Proof. The lower bound of $\left|\mathcal{N}_{\beta}\right|$ is a special case of [Lü 2008, Theorem 1.2]. Thus, it suffices to give the proof of the upper bound. For this, we merely need to show that there is a nonzero element $\beta^{\prime} \in \mathfrak{M}_{n}$ such that $n !\left|\mathcal{N}_{\beta^{\prime}}\right|=2^{n(n-1) / 2} \prod_{i=1}^{n}\left(2^{i}-1\right)$.

Consider the standard $\left(\mathbb{Z}_{2}\right)^{n}$-action $\left(\mathbb{R} P^{n}, T_{0}\right)$ of $\left(\mathbb{Z}_{2}\right)^{n}$ on the real $n$-dimensional projective space $\mathbb{R} P^{n}$ defined by $n$ commuting involutions

$$
t_{i}:\left(\left[x_{0}, x_{1}, \ldots, x_{n}\right]\right)=\left[x_{0}, x_{1}, \ldots, x_{i-1},-x_{i}, x_{i+1}, \ldots, x_{n}\right] \text { for } i=1, \ldots, n,
$$


where $t_{1}, \ldots, t_{n}$ generate $\left(\mathbb{Z}_{2}\right)^{n}$. This action fixes the $n+1$ isolated points $p_{i+1}=$ $[0, \ldots, 0,1,0, \ldots, 0]$ in which the 1 appears in the $(i+1)$-entry. One easily sees that its tangent matrix set is

$$
\mathcal{N}_{0}=\left\{\left[\Delta_{i}\right] \mid i=0,1,2, \ldots, n\right\},
$$

where $\Delta_{i}$ is $E$ when $i=0$ and for $i=1, \ldots, n$ is obtained from $E$ by replacing the $i$-th row with a row of ones. Each $\left[\Delta_{i}\right]$ corresponds to the isolated point $p_{i+1}$. Obviously, $\mathcal{N}_{0}$ is prime. By direct computations, one has that $\Delta_{i} \Delta_{i}=E$ and the result of the product $\Delta_{i} \Delta_{j}$ for $i, j \neq 0$ and $j \neq i$ just exchanges the $i$-th and $j$-th columns of $\Delta_{j}$. Thus, for $i, j \neq 0$, one has

$$
\left[\Delta_{i} \Delta_{j}\right]= \begin{cases}{[E]} & \text { if } i=j \\ {\left[\Delta_{j}\right]} & \text { if } i \neq j .\end{cases}
$$

Now, let

$B_{n+1}=\left\{\sigma \in \operatorname{GL}\left(n, \mathbb{Z}_{2}\right) \mid \sigma \mathcal{N}_{0}=\mathcal{N}_{0}\right\}, \quad$ where $\sigma \mathcal{N}_{0}=\left\{\left[\sigma \Delta_{0}\right],\left[\sigma \Delta_{1}\right], \ldots,\left[\sigma \Delta_{n}\right]\right\}$.

Obviously, $B_{n+1}$ is a subgroup of $\operatorname{GL}\left(n, \mathbb{Z}_{2}\right)$, and each element of $B_{n+1}$ actually makes a permutation in $\left[\Delta_{0}\right],\left[\Delta_{1}\right], \ldots,\left[\Delta_{n}\right]$. One then knows from (2-1) that each $\Delta_{i} \in B_{n+1}$.

Claim 1. $\left|B_{n+1}\right|=(n+1)$ !.

First, we prove that $B_{n+1}$ contains the symmetric group $\mathbf{S}_{n}$. Actually, this is because for any $E_{i j}$ in $\mathbf{S}_{n}$ and any $\Delta_{l}$,

$$
\left[E_{i j} \Delta_{l}\right]= \begin{cases}{\left[\Delta_{l}\right]} & \text { if } i, j \neq l \text { or } l=0, \\ {\left[\Delta_{i}\right]} & \text { if } j=l \neq 0, \\ {\left[\Delta_{j}\right]} & \text { if } i=l \neq 0 .\end{cases}
$$

Next, it is easy to see that any $\sigma$ in $B_{n+1}$ can be expressed as a product of some matrices of $\mathbf{S}_{n}$ and of the $\Delta_{i}$. Obviously, $\Delta_{i} \notin \mathbf{S}_{n}$ when $i \neq 0$. Thus, $B_{n+1}$ is generated by those matrices of $\mathbf{S}_{n}$ and all $\Delta_{i}$, and Claim 1 then follows from this.

Claim 2. For any $\sigma, \tau \in \operatorname{GL}\left(n, \mathbb{Z}_{2}\right)$, the set $\sigma \mathcal{N}_{0} \cap \tau \mathcal{N}_{0}$ is nonempty if and only if $\sigma \mathcal{N}_{0}=\tau \mathcal{N}_{0}$.

It is obvious that $\sigma \mathcal{N}_{0} \cap \tau \mathcal{N}_{0}$ is nonempty if $\sigma \mathcal{N}_{0}=\tau \mathcal{N}_{0}$. Conversely, if that intersection is nonempty, then there are $\left[\Delta_{i}\right],\left[\Delta_{j}\right] \in \mathcal{N}_{0}$ such that $\left[\sigma \Delta_{j}\right]=\left[\tau \Delta_{i}\right]$. By the definition of $B_{n+1}$, one has that

$$
\sigma \mathcal{N}_{0}=\tau \mathcal{N}_{0} \quad \text { if and only if } \quad \sigma^{-1} \tau \in B_{n+1} .
$$

Hence, it suffices to show that $\sigma^{-1} \tau \in B_{n+1}$. From $\left[\sigma \Delta_{j}\right]=\left[\tau \Delta_{i}\right]$, there is an element $s \in \mathbf{S}_{n}$ such that $\sigma \Delta_{j} s=\tau \Delta_{i}$, so $\sigma^{-1} \tau=\Delta_{j} s \Delta_{i}$. Note that $\Delta_{i}^{-1}=\Delta_{i}$. One also concludes that $\sigma^{-1} \tau \in B_{n+1}$. 
For any automorphism $\sigma:\left(\mathbb{Z}_{2}\right)^{n} \rightarrow\left(\mathbb{Z}_{2}\right)^{n}$ where $\sigma \in \operatorname{GL}\left(n, \mathbb{Z}_{2}\right)$, one obtains new generators $\sigma\left(t_{1}\right), \ldots, \sigma\left(t_{n}\right)$ of $\left(\mathbb{Z}_{2}\right)^{n}$, and then one obtains a new $\left(\mathbb{Z}_{2}\right)^{n}$-action $\left(\mathbb{R} P^{n}, \sigma T_{0}\right)$ from $\left(\mathbb{R} P^{n}, T_{0}\right)$ by using generators $\sigma\left(t_{1}\right), \ldots, \sigma\left(t_{n}\right)$ such that its tangent matrix set is $\left(\sigma^{-1}\right)^{\top} \mathcal{N}_{0}$. By the arguments above together with Corollary 2.2, up to equivariant cobordism there are

$$
\frac{\left|\mathrm{GL}\left(n, \mathbb{Z}_{2}\right)\right|}{\left|B_{n+1}\right|}=\frac{2^{n(n-1) / 2} \prod_{i=1}^{n}\left(2^{i}-1\right)}{(n+1) !}
$$

different $\left(\mathbb{Z}_{2}\right)^{n}$-actions $\left(\mathbb{R} P^{n}, \sigma T_{0}\right)$; specifically, the union of their tangent matrix sets just consists of all elements of $\operatorname{GL}\left(n, \mathbb{Z}_{2}\right) / \mathbf{S}_{n}$. Therefore, taking

$$
\left(T^{\prime}, M^{\prime n}\right)=\bigsqcup_{\left\{\left(\sigma^{-1}\right)^{\top}\right\} \in \mathrm{GL}\left(n, \mathbb{Z}_{2}\right) / B_{n+1}}\left(\mathbb{R} P^{n}, \sigma T_{0}\right),
$$

the tangent representation set of this action is prime, and it has

$$
(n+1) \cdot \frac{2^{n(n-1) / 2} \prod_{i=1}^{n}\left(2^{i}-1\right)}{(n+1) !}=\frac{2^{n(n-1) / 2} \prod_{i=1}^{n}\left(2^{i}-1\right)}{n !}
$$

elements. This completes the proof of the upper bound.

\section{Essential generators of $\mathfrak{M}_{n}$}

Definition 3.1. Let $\beta \neq 0$ in $\mathfrak{M}_{n}$. One says that $\beta$ is an essential generator if $\left|\mathcal{N}_{\beta+\gamma}\right| \geq\left|\mathcal{N}_{\beta}\right|$ for any $\gamma \in \mathfrak{M}_{n}$ with $\left|\mathcal{N}_{\gamma}\right|<\left|\mathcal{N}_{\beta}\right|$.

We know from Proposition 2.3 that up to equivariant cobordism there are

$$
\frac{2^{n(n-1) / 2} \prod_{i=1}^{n}\left(2^{i}-1\right)}{(n+1) !}
$$

different $\left(\mathbb{Z}_{2}\right)^{n}$-actions $\left(\mathbb{R} P^{n}, \sigma T_{0}\right)$ for $\sigma \in \mathrm{GL}\left(n, \mathbb{Z}_{2}\right)$, and each $\left(\mathbb{R} P^{n}, \sigma T_{0}\right)$ fixes just $n+1$ isolated points with different representations. Since the lower bound of $\left|\mathcal{N}_{\beta}\right|$ for any nonzero element $\beta$ of $\mathfrak{M}_{n}$ is $n+1$, each $\left(\mathbb{R} P^{n}, \sigma T_{0}\right)$ is an essential generator.

Lemma 3.1. Let $\beta \in \mathfrak{M}_{n}$. If $\beta$ is an essential generator, then

$$
\left|\mathcal{N}_{\beta}\right| \leq \begin{cases}\frac{2^{n(n-1) / 2} \prod_{i=1}^{n}\left(2^{i}-1\right)}{2 n !} & \text { if } n \text { is odd, } \\ \frac{2^{n(n-1) / 2} \prod_{i=1}^{n}\left(2^{i}-1\right)}{2(n-1) !(n+1)} & \text { if } n \text { is even. }\end{cases}
$$

Proof. The lemma is obvious if $\left|\mathcal{N}_{\beta}\right|=n+1$, so suppose that $\left|\mathcal{N}_{\beta}\right|>n+1$. Then $\beta$ is not just one of the $\left(\mathbb{R} P^{n}, \sigma T_{0}\right)$ for $\sigma \in \operatorname{GL}\left(n, \mathbb{Z}_{2}\right)$. Then one claims that for each $\left(\mathbb{Z}_{2}\right)^{n}$-action $\left(\mathbb{R} P^{n}, \sigma T_{0}\right), \mathcal{N}_{\beta}$ cannot contain more than $[(n+1) / 2]$ elements 
in $\mathcal{N}_{\left(\mathbb{R} P^{n}, \sigma T_{0}\right)}$. Actually, if not, then one has that $\left|\mathcal{N}_{\beta}\right|>\left|\mathcal{N}_{\beta+\left[\left(\mathbb{R} P^{n}, \sigma T_{0}\right)\right]}\right|$, which is impossible since $\beta$ is an essential generator. The lemma follows.

Proposition 3.2. Let $\beta \in \mathfrak{M}_{n}$. Then $\beta$ is a sum of essential generators.

Proof. The proof is trivial if $\beta=0$ or $\beta$ is an essential generator. Suppose $\beta$ is nonzero and is not an essential generator. Then there exists some element $\gamma \in \mathfrak{M}_{n}$ with $\left|\mathcal{N}_{\gamma}\right|<\left|\mathcal{N}_{\beta}\right|$ such that $\beta=(\beta+\gamma)+\gamma$ with $\left|\mathcal{N}_{\beta+\gamma}\right|<\left|\mathcal{N}_{\beta}\right|$. We repeat the argument replacing $\beta$ by $\gamma$ or $\beta+\gamma$; since $\mathfrak{M}_{n}$ is finite, the proof is ended.

\section{Small covers}

An $n$-dimensional convex polytope $P^{n}$ is said to be simple if exactly $n$ faces of codimension one meet at each of its vertices. Each point of a simple convex polytope $P^{n}$ has a neighborhood that is affine isomorphic to an open subset of the positive cone $\mathbb{R}_{\geq 0}^{n}$. A smooth closed $n$-manifold $M^{n}$ is said to be a small cover if it admits an effective smooth $\left(\mathbb{Z}_{2}\right)^{n}$-action that is locally isomorphic to the standard action of $\left(\mathbb{Z}_{2}\right)^{n}$ on $\mathbb{R}^{n}$ such that the orbit space of the action is a simple convex polytope $P^{n}$.

A small cover is a special 2-torus manifold. A canonical example of small cover is the $n$-dimensional real projective space $\mathbb{R} P^{n}$ with the standard $\left(\mathbb{Z}_{2}\right)^{n}$ action whose orbit space is the $n$-simplex $\Delta^{n}$.

Suppose that $\pi: M^{n} \rightarrow P^{n}$ is a small cover over a simple convex polytope $P^{n}$. Let $\mathscr{F}\left(P^{n}\right)=\left\{F_{1}, \ldots, F_{\ell}\right\}$ be the set of codimension one faces (facets) of $P^{n}$. Then there are $\ell$ connected submanifolds $M_{1}, \ldots, M_{\ell}$ determined by $\pi$ and $F_{i}$ (that is, $M_{i}=\pi^{-1}\left(F_{i}\right)$ ), which are called characteristic submanifolds here. Each submanifold $M_{i}$ is fixed pointwise by a $\mathbb{Z}_{2}$-subgroup $G_{i}$ of $\left(\mathbb{Z}_{2}\right)^{n}$, so that each facet $F_{i}$ corresponds to the $\mathbb{Z}_{2}$-subgroup $G_{i}$. Since there is a canonical isomorphism from $\left(\mathbb{Z}_{2}\right)^{n}$ to $\operatorname{Hom}\left(\mathbb{Z}_{2},\left(\mathbb{Z}_{2}\right)^{n}\right)$, each $\mathbb{Z}_{2}$-subgroup $G_{i}$ corresponds to an element $v_{i}$ in $\operatorname{Hom}\left(\mathbb{Z}_{2},\left(\mathbb{Z}_{2}\right)^{n}\right)$. For each face $F$ of codimension $s$, since $P^{n}$ is simple, there are $s$ facets $F_{i_{1}}, \ldots, F_{i_{s}}$ whose intersection is $F$. Then the corresponding characteristic submanifolds $M_{i_{1}}, \ldots, M_{i_{s}}$ intersect transversally in the $(n-s)$-dimensional submanifold $\pi^{-1}(F)$, and the isotropy subgroup $G_{F}$ of $\pi^{-1}(F)$ is a subtorus of rank $s$ and is generated by $G_{i_{1}}, \ldots, G_{i_{s}}$ (or is determined by $v_{i_{1}}, \ldots, v_{i_{s}}$ in $\left.\operatorname{Hom}\left(\mathbb{Z}_{2},\left(\mathbb{Z}_{2}\right)^{n}\right)\right)$. Thus, this actually gives a characteristic function [Davis and Januszkiewicz 1991]

$$
\lambda: \mathscr{F}\left(P^{n}\right) \rightarrow \operatorname{Hom}\left(\mathbb{Z}_{2},\left(\mathbb{Z}_{2}\right)^{n}\right), \quad F_{i} \mapsto v_{i}
$$

such that for any face $F=F_{i_{1}} \cap \cdots \cap F_{i_{s}}$ of $P^{n}, \lambda\left(F_{i_{1}}\right), \ldots, \lambda\left(F_{i_{s}}\right)$ are linearly independent in $\operatorname{Hom}\left(\mathbb{Z}_{2},\left(\mathbb{Z}_{2}\right)^{n}\right)$. When $\operatorname{dim} F=0$ (that is, $\left.s=n\right), F$ is a vertex of $P^{n}$, which corresponds to a $\left(\mathbb{Z}_{2}\right)^{n}$-fixed point $p$ of $M$. In this case, $\lambda\left(F_{i_{1}}\right), \ldots, \lambda\left(F_{i_{n}}\right)$ uniquely determines a dual basis of $\operatorname{Hom}\left(\left(\mathbb{Z}_{2}\right)^{n}, \mathbb{Z}_{2}\right)$, which just gives the tangent 
representation at $p$. Thus, the characteristic function $\lambda$ completely determines the tangent representation set $\mathcal{N}_{(M, \phi)}$ of fixed points of $M^{n}$, where $\phi$ denotes the $\left(\mathbb{Z}_{2}\right)^{n}$ action on $M$.

By [Davis and Januszkiewicz 1991], there is a reconstruction process of $M^{n}$ by using the product bundle $\left(\mathbb{Z}_{2}\right)^{n} \times P^{n}$ and $\lambda$. Note that each point $q \in \partial P^{n}$ must lie in the relative interior of a unique face $F(q)$ of $P^{n}$. Then, one may define an equivalence relation on $\left(\mathbb{Z}_{2}\right)^{n} \times P^{n}$ by

$$
\left(t_{1}, x\right) \sim\left(t_{2}, x\right) \text { if and only if } t_{1}^{-1} t_{2} \in G_{F(q)},
$$

where $x \in F(q)$; then the quotient space

$$
M(\lambda):=\left(\mathbb{Z}_{2}\right)^{n} \times P^{n} /\left(t_{1}, x\right) \sim\left(t_{2}, x\right)
$$

is equivariantly homeomorphic to $M^{n}$. Obviously, both $M^{n}$ and $M(\lambda)$ have the same characteristic function, so they also are cobordant equivariantly.

We denote by $\Lambda\left(P^{n}\right)$ the set of all characteristic functions on $P^{n}$.

Proposition 4.1. Let $\pi: M^{n} \rightarrow P^{n}$ be a small cover over a simple convex polytope $P^{n}$. Then all small covers over $P^{n}$ are given by $\left\{M(\lambda) \mid \lambda \in \Lambda\left(P^{n}\right)\right\}$.

Remark. Generally speaking, one cannot make sure that there always exist characteristic functions (or colorings) over a simple convex polytope $P^{n}$ when $n \geq 4$. For example, see [Davis and Januszkiewicz 1991, Nonexamples 1.22]. However, the Four Color Theorem ensures that every 3-dimensional simple convex polytope admits characteristic functions.

The correspondence $\lambda \mapsto \sigma \circ \lambda$ defines an action of $\operatorname{GL}\left(n, \mathbb{Z}_{2}\right)$ on $\Lambda\left(P^{n}\right)$, and it then induces an action of $\operatorname{GL}\left(n, \mathbb{Z}_{2}\right)$ on $\left\{M(\lambda) \mid \lambda \in \Lambda\left(P^{n}\right)\right\}$ that takes $M(\lambda)$ to $M(\sigma \circ \lambda)$. It is easy to check that these two actions are free.

The following two kinds of small covers play an important role in determining of the structure of $\mathfrak{M}_{3}$.

Example 4.1 (small covers over a 3-complex $\Delta^{3}$ ). A 3-simplex $\Delta^{3}$ has four 2faces, and a canonical characteristic function $\lambda_{0}$ on it is defined by assigning to $\rho_{1}^{*}$, $\rho_{2}^{*}, \rho_{3}^{*}, \rho_{1}^{*}+\rho_{2}^{*}+\rho_{3}^{*}$ the four 2-faces of $\Delta^{3}$, where $\left\{\rho_{1}^{*}, \rho_{2}^{*}, \rho_{3}^{*}\right\}$ is the standard basis of $\operatorname{Hom}\left(\mathbb{Z}_{2},\left(\mathbb{Z}_{2}\right)^{3}\right)$, corresponding to $\rho_{1}, \rho_{2}, \rho_{3}$ of $\operatorname{Hom}\left(\left(\mathbb{Z}_{2}\right)^{3}, \mathbb{Z}_{2}\right)$. Thus, $\left\{\sigma \circ \lambda_{0} \mid\right.$ $\left.\sigma \in \mathrm{GL}\left(3, \mathbb{Z}_{2}\right)\right\}$ gives all characteristic functions on $\Delta^{3}$. Since the characteristic function of the standard action $T_{0}$ of $\left(\mathbb{Z}_{2}\right)^{3}$ on $\mathbb{R} P^{3}$ is just $\lambda_{0}$,

$$
\left\{M\left(\sigma \circ \lambda_{0}\right) \mid \sigma \in \operatorname{GL}\left(3, \mathbb{Z}_{2}\right)\right\}=\left\{\left(\mathbb{R} P^{3}, \sigma T_{0}\right) \mid \sigma \in \operatorname{GL}\left(3, \mathbb{Z}_{2}\right)\right\} .
$$

Proposition 2.3 shows that up to equivariant cobordism, this set has 7 different denoted by $\left(\mathbb{R} P^{3}, T_{0}\right),\left(\mathbb{R} P^{3}, T_{1}\right), \ldots,\left(\mathbb{R} P^{3}, T_{6}\right)$. A direct calculation gives the Table I, which lists the tangent representation sets of these seven small covers. 


\begin{tabular}{ll}
\hline small cover $M$ & tangent representation set $\mathcal{N}_{M}$ \\
\hline $\left.\mathbb{R} P^{3}, T_{0}\right)$ & $\rho_{1} \rho_{2} \rho_{3}, \rho_{1}\left(\rho_{1}+\rho_{2}\right)\left(\rho_{1}+\rho_{3}\right)$, \\
& $\rho_{2}\left(\rho_{1}+\rho_{2}\right)\left(\rho_{2}+\rho_{3}\right), \rho_{3}\left(\rho_{1}+\rho_{3}\right)\left(\rho_{2}+\rho_{3}\right)$ \\
$\left(\mathbb{R} P^{3}, T_{1}\right)$ & $\rho_{1}\left(\rho_{1}+\rho_{2}\right)\left(\rho_{1}+\rho_{2}+\rho_{3}\right), \rho_{1} \rho_{2}\left(\rho_{2}+\rho_{3}\right)$, \\
& $\rho_{2} \rho_{3}\left(\rho_{1}+\rho_{2}\right), \rho_{3}\left(\rho_{2}+\rho_{3}\right)\left(\rho_{1}+\rho_{2}+\rho_{3}\right)$ \\
$\left(\mathbb{R} P^{3}, T_{2}\right)$ & $\rho_{1}\left(\rho_{1}+\rho_{3}\right)\left(\rho_{1}+\rho_{2}+\rho_{3}\right), \rho_{1} \rho_{3}\left(\rho_{2}+\rho_{3}\right)$, \\
& $\rho_{2} \rho_{3}\left(\rho_{1}+\rho_{3}\right), \rho_{2}\left(\rho_{2}+\rho_{3}\right)\left(\rho_{1}+\rho_{2}+\rho_{3}\right)$ \\
$\left(\mathbb{R} P^{3}, T_{3}\right)$ & $\rho_{2}\left(\rho_{1}+\rho_{2}\right)\left(\rho_{1}+\rho_{2}+\rho_{3}\right), \rho_{1} \rho_{2}\left(\rho_{1}+\rho_{3}\right)$, \\
& $\rho_{1} \rho_{3}\left(\rho_{1}+\rho_{2}\right), \rho_{3}\left(\rho_{1}+\rho_{3}\right)\left(\rho_{1}+\rho_{2}+\rho_{3}\right)$ \\
$\left(\mathbb{R} P^{3}, T_{4}\right)$ & $\rho_{1}\left(\rho_{1}+\rho_{2}\right)\left(\rho_{2}+\rho_{3}\right), \rho_{1} \rho_{2}\left(\rho_{1}+\rho_{2}+\rho_{3}\right)$, \\
& $\rho_{2}\left(\rho_{1}+\rho_{2}\right)\left(\rho_{1}+\rho_{3}\right),\left(\rho_{1}+\rho_{3}\right)\left(\rho_{2}+\rho_{3}\right)\left(\rho_{1}+\rho_{2}+\rho_{3}\right)$ \\
$\left(\mathbb{R} P^{3}, T_{5}\right)$ & $\rho_{1}\left(\rho_{1}+\rho_{3}\right)\left(\rho_{2}+\rho_{3}\right), \rho_{1} \rho_{3}\left(\rho_{1}+\rho_{2}+\rho_{3}\right)$, \\
& $\rho_{3}\left(\rho_{1}+\rho_{2}\right)\left(\rho_{1}+\rho_{3}\right),\left(\rho_{1}+\rho_{2}\right)\left(\rho_{2}+\rho_{3}\right)\left(\rho_{1}+\rho_{2}+\rho_{3}\right)$ \\
$\left(\mathbb{R} P^{3}, T_{6}\right)$ & $\rho_{2}\left(\rho_{1}+\rho_{3}\right)\left(\rho_{2}+\rho_{3}\right), \rho_{2} \rho_{3}\left(\rho_{1}+\rho_{2}+\rho_{3}\right)$, \\
& $\left(\rho_{1}+\rho_{2}\right)\left(\rho_{1}+\rho_{3}\right)\left(\rho_{1}+\rho_{2}+\rho_{3}\right), \rho_{3}\left(\rho_{1}+\rho_{2}\right)\left(\rho_{2}+\rho_{3}\right)$ \\
\hline
\end{tabular}

\section{Table I}

Example 4.2 (small covers over a prism $P^{3}$ ). There exists only one simple convex 3 -polytope with six vertices (that is, a prism $P^{3}$ ); see [Ewald 1996]. Let $F_{1}, F_{2}, F_{4}$ denote the three square facets, and $F_{3}, F_{5}$ the two triangular facets in $P^{3}$. From [Cai et al. 2007] we know that essentially there are five different characteristic functions $\lambda_{1}, \lambda_{2}, \lambda_{3}, \lambda_{4}, \lambda_{5}$ under the action of $\operatorname{GL}\left(3, \mathbb{Z}_{2}\right)$ on $\Lambda\left(P^{3}\right)$, which are defined by this table:

\begin{tabular}{l|lllll} 
& $F_{1}$ & $F_{2}$ & $F_{3}$ & $F_{4}$ & $F_{5}$ \\
\hline$\lambda_{1}$ & $\rho_{1}^{*}$ & $\rho_{2}^{*}$ & $\rho_{3}^{*}$ & $\rho_{1}^{*}+\rho_{2}^{*}$ & $\rho_{1}^{*}+\rho_{2}^{*}+\rho_{3}^{*}$ \\
$\lambda_{2}$ & $\rho_{1}^{*}$ & $\rho_{2}^{*}$ & $\rho_{3}^{*}$ & $\rho_{1}^{*}+\rho_{2}^{*}$ & $\rho_{1}^{*}+\rho_{3}^{*}$ \\
$\lambda_{3}$ & $\rho_{1}^{*}$ & $\rho_{2}^{*}$ & $\rho_{3}^{*}$ & $\rho_{1}^{*}+\rho_{2}^{*}$ & $\rho_{2}^{*}+\rho_{3}^{*}$ \\
$\lambda_{4}$ & $\rho_{1}^{*}$ & $\rho_{2}^{*}$ & $\rho_{3}^{*}$ & $\rho_{1}^{*}+\rho_{2}^{*}$ & $\rho_{3}^{*}$ \\
$\lambda_{5}$ & $\rho_{1}^{*}$ & $\rho_{2}^{*}$ & $\rho_{3}^{*}$ & $\rho_{1}^{*}+\rho_{2}^{*}+\rho_{3}^{*}$ & $\rho_{3}^{*}$
\end{tabular}

It is easy to check that for any $\sigma \in \mathrm{GL}\left(3, \mathbb{Z}_{2}\right)$, every one of $M\left(\sigma \circ \lambda_{4}\right)$ and $M\left(\sigma \circ \lambda_{5}\right)$ always bounds equivariantly. A direct calculation shows that

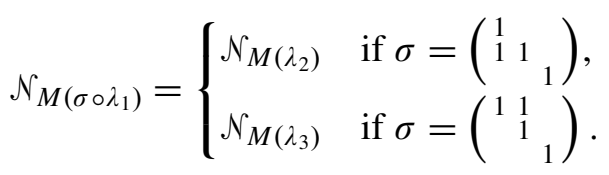

Since $\mathcal{N}_{M\left(\lambda_{1}\right)}$ is prime, we know by Corollary 2.2 that if $\beta \in \mathfrak{M}_{3}$ is represented by a small cover over $P^{3}$, then $\beta$ belongs to the set of equivariant cobordism classes 
coming from the set $\left\{M\left(\sigma \circ \lambda_{1}\right) \mid \sigma \in \mathrm{GL}\left(3, \mathbb{Z}_{2}\right)\right\}$. By further computations, one obtains that there are only four matrices,

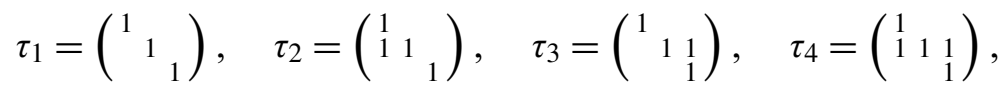

such that $\tau_{i} \mathcal{N}_{M\left(\lambda_{1}\right)}=\mathcal{N}_{M\left(\lambda_{1}\right)}$ for $i=1,2,3,4$, and these four matrices form a subgroup of $\operatorname{GL}\left(3, \mathbb{Z}_{2}\right)$. Thus, up to equivariant cobordism, there are $\left|\operatorname{GL}\left(3, \mathbb{Z}_{2}\right)\right| / 4=$ 42 different nonbounding small covers over $P^{3}$. We can even construct such small covers as follows. Consider the $\left(\mathbb{Z}_{2}\right)^{3}$-action $\Phi_{0}$ on $S^{1} \times \mathbb{R} P^{2}=S^{1} \times \mathbb{R} P(\mathbb{C} \oplus \mathbb{R})$ defined by the three commutative involutions

$$
\begin{aligned}
& t_{1}:(z,[v, w]) \mapsto(\bar{z},[\bar{z} v, w]), \\
& t_{2}:(z,[v, w]) \mapsto(z,[z \bar{v}, w]), \\
& t_{3}:(z,[v, w]) \mapsto(z,[-z \bar{v}, w]) .
\end{aligned}
$$

This action fixes the six isolated points $( \pm 1,[0,1]),( \pm 1,[1,0]),( \pm 1,[\sqrt{-1}, 0])$, and its orbit space is just a prism $P^{3}$. A direct calculation shows that $\mathcal{N}_{\left(S^{1} \times \mathbb{R} P^{2}, \Phi_{0}\right)}$ consists of the six distinct monomials

$$
\begin{array}{lll}
\rho_{1} \rho_{2} \rho_{3}, & \rho_{1} \rho_{2}\left(\rho_{2}+\rho_{3}\right), & \rho_{1} \rho_{3}\left(\rho_{2}+\rho_{3}\right), \\
\rho_{1}\left(\rho_{1}+\rho_{2}\right)\left(\rho_{1}+\rho_{3}\right), & \rho_{1}\left(\rho_{1}+\rho_{2}\right)\left(\rho_{2}+\rho_{3}\right), & \rho_{1}\left(\rho_{1}+\rho_{3}\right)\left(\rho_{2}+\rho_{3}\right)
\end{array}
$$

of $\mathbb{Z}_{2}\left[\rho_{1}, \rho_{2}, \rho_{3}\right]$, so $\left(S^{1} \times \mathbb{R} P^{2}, \Phi_{0}\right)$ is nonbounding. Further, up to equivariant cobordism, 42 different nonbounding small covers over $P^{3}$ can be given by applying automorphisms of $\left(\mathbb{Z}_{2}\right)^{3}$ on $\left(S^{1} \times \mathbb{R} P^{2}, \Phi_{0}\right)$, and they are denoted by $\left(S^{1} \times \mathbb{R} P^{2}, \Phi_{0}\right),\left(S^{1} \times \mathbb{R} P^{2}, \Phi_{1}\right), \ldots,\left(S^{1} \times \mathbb{R} P^{2}, \Phi_{41}\right)$.

\section{Graphs of actions}

Given a nonzero element $\beta$ in $\mathfrak{M}_{n}$, let $\left(M^{n}, \phi\right)$ be a representative of $\beta$ such that $\mathcal{N}_{(M, \phi)}$ is prime. For a nontrivial irreducible representation $\rho$ in $\operatorname{Hom}\left(\left(\mathbb{Z}_{2}\right)^{n}, \mathbb{Z}_{2}\right)$, let $C$ be a component of the fixed point set of $\operatorname{ker} \rho\left(\cong\left(\mathbb{Z}_{2}\right)^{n-1}\right)$ acting on $M$, such that $\operatorname{dim} C>0$ and the action of $\left(\mathbb{Z}_{2}\right)^{n} / \operatorname{ker} \rho$ on $C$ has a nonempty fixed point set. Then the dimension of $C$ must be 1 since the action is effective, and thus $C$ is equivariantly diffeomorphic to the circle $S^{1}$ with a reflection fixing just two fixed points. Then one has an edge joining these two fixed points, which is labeled by $\rho$. Furthermore, one can obtain a graph $\Gamma_{(M, \phi)}$, which is the union of all those edges chosen for each $\rho$ and $C$. Clearly, the set of vertices of $\Gamma_{(M, \phi)}$ is just the fixed point set of $\left(\mathbb{Z}_{2}\right)^{n}$ acting on $M$. Since the tangent representation at a fixed point $p$ has $n$ irreducible summands, the number of edges in $\Gamma_{(M, \phi)}$ meeting at $p$ is exactly $n$, so $\Gamma_{(M, \phi)}$ is a regular graph of valence $n$. It should be pointed out 
that, generally, $\Gamma_{(M, \phi)}$ is not determined by $\beta$ uniquely, and it depends upon the choice of representatives of $\beta$.

Let $E\left(\Gamma_{(M, \phi)}\right)$ denote the set of all edges in $\Gamma_{(M, \phi)}$, and let $V\left(\Gamma_{(M, \phi)}\right)$ denote the vertices in $\Gamma_{(M, \phi)}$. Given a vertex $p$ in $V\left(\Gamma_{(M, \phi)}\right)$, let $E_{p}$ denote the set of $n$ edges joining to $p$. Then there is a natural map $\alpha: E\left(\Gamma_{(M, \phi)}\right) \rightarrow \operatorname{Hom}\left(\left(\mathbb{Z}_{2}\right)^{n}, \mathbb{Z}_{2}\right)$, called an axial function or a $\left(\mathbb{Z}_{2}\right)^{n}$-coloring; see [Guillemin and Zara 2001; 2003; Bao and Lü 2008]. One knows from [Lü 2008] that $\alpha$ satisfies the following properties:

(1) $\alpha\left(E_{p}\right)$ spans $\operatorname{Hom}\left(\left(\mathbb{Z}_{2}\right)^{n}, \mathbb{Z}_{2}\right)$ for each vertex $p$ in $V\left(\Gamma_{(M, \phi)}\right)$,

(2) for each edge $e$ in $E_{\Gamma_{(M, \phi)}}$,

$$
\prod_{x \in E_{p}-E_{e}} \alpha(x) \equiv \prod_{y \in E_{q}-E_{e}} \alpha(y) \quad \bmod \alpha(e),
$$

where $p$ and $q$ are the two endpoints of $e$, and $E_{e}$ denotes the set of all edges joining the two endpoints of $e$. The pair $\left(\Gamma_{(M, \phi)}, \alpha\right)$ is called the moment graph of $\left(M^{n}, \phi\right)$. Since $\mathcal{N}_{(M, \phi)}$ is prime, one has from [Lü 2008] that $\left|E_{e}\right|=1$ for each edge $e$ in $\Gamma_{(M, \phi)}$.

Note. If $M$ is a small cover over a simple convex polytope $P^{n}$, then $\Gamma_{(M, \phi)}$ is just the 1-skeleton of $P^{n}$. In this case, it is easy to see that the map $\alpha: E_{\Gamma_{(M, \phi)}} \rightarrow$ $\operatorname{Hom}\left(\left(\mathbb{Z}_{2}\right)^{n}, \mathbb{Z}_{2}\right)$ is dual to the characteristic function $\lambda: \mathscr{F}\left(P^{n}\right) \rightarrow \operatorname{Hom}\left(\mathbb{Z}_{2},\left(\mathbb{Z}_{2}\right)^{n}\right)$. In other words, both $\alpha$ and $\lambda$ are determined by each other.

By [Bao and Lü 2008] we know that $\left(\Gamma_{(M, \phi)}, \alpha\right)$ is a "good" $\left(\mathbb{Z}_{2}\right)^{n}$-coloring, so that each $k$-nest $\Delta^{k}$ of $\left(\Gamma_{(M, \phi)}, \alpha\right)$ is a connected regular $k$-valent subgraph of $\Gamma_{(M, \phi)}$ with $\operatorname{dim} \operatorname{Span} \alpha\left(\Delta^{k}\right)=k$, where $\operatorname{Span} \alpha\left(\Delta^{k}\right)$ denotes the linear space spanned by all colors of edges in $\Delta^{k}$. By $\mathscr{K}_{\left(\Gamma_{(M, \phi)}, \alpha\right)}$ one denotes the set of all nests of $\left(\Gamma_{(M, \phi)}, \alpha\right)$. Since each $k$-nest $(k>0)$ determines a $k$-dimensional subspace of $\operatorname{Hom}\left(\left(\mathbb{Z}_{2}\right)^{n}, \mathbb{Z}_{2}\right)$, it corresponds to an $(n-k)$-dimensional subspace in the dual space $\operatorname{Hom}\left(\mathbb{Z}_{2},\left(\mathbb{Z}_{2}\right)^{n}\right)$. This actually gives a dual map $\eta$ from $\mathscr{K}_{\left(\Gamma_{(M, \phi)}, \alpha\right)}$ to the set of all subspaces of $\operatorname{Hom}\left(\mathbb{Z}_{2},\left(\mathbb{Z}_{2}\right)^{n}\right)$, which is just the characteristic function when $M$ is a small cover. Obviously, $\eta$ maps each $(n-1)$-dimensional nest of $\mathscr{K}_{\left(\Gamma_{(M, \phi)}, \alpha\right)}$ to a nonzero element in $\operatorname{Hom}\left(\mathbb{Z}_{2},\left(\mathbb{Z}_{2}\right)^{n}\right)$. Since each vertex $p$ is the intersection of $n(n-1)$-nests of $\mathscr{K}_{\left(\Gamma_{(M, \phi)}, \alpha\right)}$, it corresponds to a basis of $\operatorname{Hom}\left(\mathbb{Z}_{2},\left(\mathbb{Z}_{2}\right)^{n}\right)$, which is just the dual basis of the basis $\alpha\left(E_{p}\right)$ in $\operatorname{Hom}\left(\left(\mathbb{Z}_{2}\right)^{n}, \mathbb{Z}_{2}\right)$.

Proposition 5.1 [Bao and Lü 2008]. If $\operatorname{dim} M=3$, then $\left(\Gamma_{(M, \phi)}, \alpha\right)$ admits a 2skeletal expansion $(N, K)$ such that $N$ is a closed surface.

We will use this result to study the group structure of $\mathfrak{M}_{3}$. Note that if $\operatorname{dim} M>4$, under what condition $\left(\Gamma_{(M, \phi)}, \alpha\right)$ admits a skeletal expansion is still open. 


\section{Determination of $\mathfrak{M}_{3}$}

By Proposition 3.2, the structure of $\mathfrak{M}_{3}$ is determined by the essential generators of $\mathfrak{M}_{3}$.

Lemma 6.1. Let $\beta \in \mathfrak{M}_{3}$. Then $\left|\mathcal{N}_{\beta}\right|$ is even.

Proof. The Euler characteristic of any 3-dimensional closed manifold is always zero. The lemma then follows from the classical Smith theorem.

The following proposition characterizes the essential generators of $\mathfrak{M}_{3}$.

Proposition 6.2. A nonzero element $\beta \in \mathfrak{M}_{3}$ is an essential generator if and only if $\left|\mathcal{N}_{\beta}\right| \leq 6$. In this case $\beta$ is represented by a small cover either of the type $\left(\mathbb{R} P^{3}, \sigma T_{0}\right)$, or of the type $\left(S^{1} \times \mathbb{R} P^{2}, \sigma \Phi_{0}\right)$, for some $\sigma \in \mathrm{GL}\left(3, \mathbb{Z}_{2}\right)$.

The lemma below proves part of this proposition.

Lemma 6.3. Let $\beta \in \mathfrak{M}_{3}$ be nonzero. If $\left|\mathcal{N}_{\beta}\right| \leq 6$, then $\beta$ is an essential generator.

Proof. If $\left|\mathcal{N}_{\beta}\right|=4$, then $\beta$ is an essential generator because the lower bound for $\left|\mathcal{N}_{\beta}\right|$ is 4 . Thus, by Lemma 6.1, it suffices to consider the case $\left|\mathcal{N}_{\beta}\right|=6$. From Example 4.1, we see that the sets $\mathcal{N}_{\left(\mathbb{R} P^{3}, T_{i}\right)}$ for $i=0,1, \ldots, 6$ are disjoint. We first claim that any intersection $\mathcal{N}_{\beta} \cap \mathcal{N}_{\left(\mathbb{R} P^{3}, T_{i}\right)}$ cannot contain four elements. If one did, then there would exist some $i^{\prime}$ such that $\left|\mathcal{N}_{\beta+\left[\left(\mathbb{R} P^{3}, T_{i^{\prime}}\right)\right]}\right|=2$. By [Kosniowski and Stong 1979], $\beta+\left[\left(\mathbb{R} P^{3}, T_{i^{\prime}}\right)\right]$ would then be zero in $\mathfrak{M}_{3}$, which is impossible. Next, we prove that any intersection $\mathcal{N}_{\beta} \cap \mathcal{N}_{\left(\mathbb{R} P^{3}, T_{i}\right)}$ cannot contain three elements. If one did, then there would be some $i^{\prime \prime}$ such that $\left|\mathcal{N}_{\beta+\left[\left(\mathbb{R} P^{3}, T_{i^{\prime \prime}}\right)\right]}\right|=4$, and so $\beta+\left[\left(\mathbb{R} P^{3}, T_{i^{\prime \prime}}\right)\right]$ would be the equivariant cobordism class of another $\left(\mathbb{R} P^{3}, T_{j}\right)$ with $j \neq i^{\prime \prime}$. Then $\beta$ would be the sum $\left[\left(\mathbb{R} P^{3}, T_{i^{\prime \prime}}\right)\right]+\left[\left(\mathbb{R} P^{3}, T_{j}\right)\right]$, so $\left|\mathcal{N}_{\beta}\right|$ would be 8 rather than 6 . This is a contradiction, and thus $\left|\mathcal{N}_{\beta} \cap \mathcal{N}_{\left(\mathbb{R} P^{3}, T_{i}\right)}\right|$ is less than 3 . The lemma follows.

The following lemma indicates the connection between

$\mathscr{A}=\left\{\left(\mathbb{R} P^{3}, T_{i}\right) \mid i=0,1, \ldots, 6\right\}$ and $\mathscr{B}=\left\{\left(S^{1} \times \mathbb{R} P^{2}, \Phi_{j}\right) \mid j=0,1, \ldots, 41\right\}$.

Lemma 6.4. Each $\left(\mathbb{R} P^{3}, T_{i}\right)$ of $\mathscr{A}$ corresponds to six small covers $\left(S^{1} \times \mathbb{R} P^{2}, \Phi_{i_{1}}\right)$, $\ldots,\left(S^{1} \times \mathbb{R} P^{2}, \Phi_{i_{6}}\right)$ of $\mathscr{B}$ in such a way that these six small covers are not cobordant to each other, and $\left|\mathcal{N}_{\left(\mathbb{R} P^{3}, T_{i}\right)} \cap \mathcal{N}_{\left(S^{1} \times \mathbb{R} P^{2}, \Phi_{i u}\right)}\right|=2$ for $u=1, \ldots, 6$.

Proof. Since all $\mathcal{N}_{\left(\mathbb{R} P^{3}, T_{i}\right)}$ for $i=0,1, \ldots, 6$ are distinct and since all $\left(\mathbb{R} P^{3}, T_{i}\right)$ for $i=0,1, \ldots, 6$, can be translated to each other up to cobordism by applying automorphisms of $\left(\mathbb{Z}_{2}\right)^{3}$, it suffices to consider the case of $\left(\mathbb{R} P^{3}, T_{0}\right)$. We see from Table I that $\mathcal{N}_{\left(\mathbb{R} P^{3}, T_{0}\right)}$ is equal to

$$
\left\{\rho_{1} \rho_{2} \rho_{3}, \rho_{1}\left(\rho_{1}+\rho_{2}\right)\left(\rho_{1}+\rho_{3}\right), \rho_{2}\left(\rho_{1}+\rho_{2}\right)\left(\rho_{2}+\rho_{3}\right), \rho_{3}\left(\rho_{1}+\rho_{3}\right)\left(\rho_{2}+\rho_{3}\right)\right\} .
$$




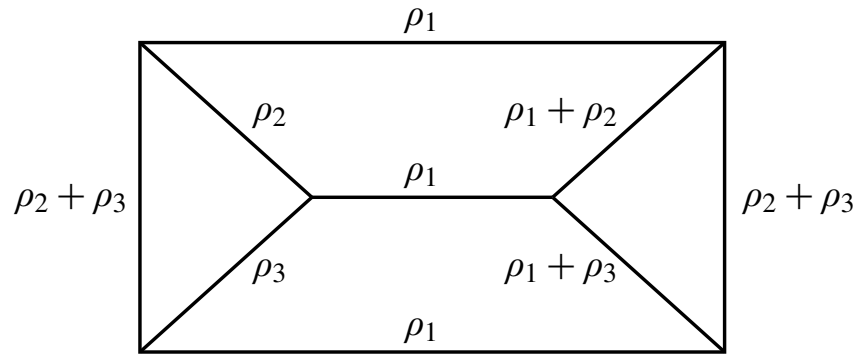

Figure 1. An axial function $\alpha$ on the 1-skeleton of a prism $P^{3}$.

Obviously, any two monomials of $\mathcal{N}_{\left(\mathbb{R} P^{3}, T_{0}\right)}$ give five elements of $\operatorname{Hom}\left(\left(\mathbb{Z}_{2}\right)^{3}, \mathbb{Z}_{2}\right)$, and there are exactly six such pairs in $\mathcal{N}_{\left(\mathbb{R} P^{3}, T_{0}\right)}$. Considering two monomials $\rho_{1} \rho_{2} \rho_{3}$ and $\rho_{1}\left(\rho_{1}+\rho_{2}\right)\left(\rho_{1}+\rho_{3}\right)$ of $\mathcal{N}_{\left(\mathbb{R} P^{3}, T_{0}\right)}$, we get five elements $\rho_{1}, \rho_{2}, \rho_{3}$, $\rho_{1}+\rho_{2}$ and $\rho_{1}+\rho_{3}$ of $\operatorname{Hom}\left(\left(\mathbb{Z}_{2}\right)^{3}, \mathbb{Z}_{2}\right)$. Using these five elements, we can define an axial function $\alpha$ on the 1-skeleton of a prism $P^{3}$ as shown in Figure 1.

Since $\alpha$ uniquely determines a characteristic function on $P^{3}$, we obtain a small cover $\left(S^{1} \times \mathbb{R} P^{2}, \Phi_{0_{1}}\right)$ with six fixed points over $P^{3}$ such that its tangent representation set $\mathcal{N}_{\left(S^{1} \times \mathbb{R} P^{2}, \Phi_{0_{1}}\right)}$ consists of six monomials $\rho_{1} \rho_{2} \rho_{3}, \rho_{1}\left(\rho_{1}+\rho_{2}\right)\left(\rho_{1}+\rho_{3}\right)$, $\rho_{1} \rho_{2}\left(\rho_{2}+\rho_{3}\right), \rho_{1} \rho_{3}\left(\rho_{2}+\rho_{3}\right), \rho_{1}\left(\rho_{1}+\rho_{2}\right)\left(\rho_{2}+\rho_{3}\right)$ and $\rho_{1}\left(\rho_{1}+\rho_{3}\right)\left(\rho_{2}+\rho_{3}\right)$. Similarly, for other five pairs in $\mathcal{N}_{\left(\mathbb{R} P^{3}, T_{0}\right)}$, we can obtain small covers $\left(S^{1} \times \mathbb{R} P^{2}, \Phi_{0_{u}}\right)$ for $u=2, \ldots, 6$ with their tangent representation sets as follows:

\begin{tabular}{cc}
\hline$u$ & $\mathcal{N}_{\left(S^{1} \times \mathbb{R} P^{2}, \Phi_{0_{u}}\right)}$ \\
\hline 2 & $\left\{\rho_{1} \rho_{2} \rho_{3}, \rho_{1} \rho_{2}\left(\rho_{1}+\rho_{3}\right), \rho_{2} \rho_{3}\left(\rho_{1}+\rho_{3}\right), \rho_{2}\left(\rho_{1}+\rho_{2}\right)\left(\rho_{2}+\rho_{3}\right)\right.$, \\
& $\left.\rho_{2}\left(\rho_{1}+\rho_{2}\right)\left(\rho_{1}+\rho_{3}\right), \rho_{2}\left(\rho_{1}+\rho_{3}\right)\left(\rho_{2}+\rho_{3}\right)\right\}$ \\
3 & $\left\{\rho_{1} \rho_{2} \rho_{3}, \rho_{1} \rho_{3}\left(\rho_{1}+\rho_{2}\right), \rho_{2} \rho_{3}\left(\rho_{1}+\rho_{2}\right), \rho_{3}\left(\rho_{1}+\rho_{3}\right)\left(\rho_{2}+\rho_{3}\right)\right.$, \\
& $\left.\rho_{3}\left(\rho_{1}+\rho_{2}\right)\left(\rho_{1}+\rho_{3}\right), \rho_{3}\left(\rho_{1}+\rho_{2}\right)\left(\rho_{2}+\rho_{3}\right)\right\}$ \\
4 & $\left\{\rho_{1}\left(\rho_{1}+\rho_{2}\right)\left(\rho_{1}+\rho_{3}\right), \rho_{1} \rho_{3}\left(\rho_{1}+\rho_{2}\right), \rho_{3}\left(\rho_{1}+\rho_{2}\right)\left(\rho_{1}+\rho_{3}\right)\right.$, \\
& $\left.\rho_{2}\left(\rho_{1}+\rho_{2}\right)\left(\rho_{2}+\rho_{3}\right), \rho_{2} \rho_{3}\left(\rho_{1}+\rho_{2}\right), \rho_{3}\left(\rho_{1}+\rho_{2}\right)\left(\rho_{2}+\rho_{3}\right)\right\}$ \\
5 & $\left\{\rho_{1}\left(\rho_{1}+\rho_{2}\right)\left(\rho_{1}+\rho_{3}\right), \rho_{1} \rho_{2}\left(\rho_{1}+\rho_{3}\right), \rho_{2}\left(\rho_{1}+\rho_{2}\right)\left(\rho_{1}+\rho_{3}\right)\right.$, \\
& $\left.\rho_{3}\left(\rho_{1}+\rho_{3}\right)\left(\rho_{2}+\rho_{3}\right), \rho_{2} \rho_{3}\left(\rho_{1}+\rho_{3}\right), \rho_{2}\left(\rho_{1}+\rho_{3}\right)\left(\rho_{2}+\rho_{3}\right)\right\}$ \\
6 & $\left\{\rho_{2}\left(\rho_{1}+\rho_{2}\right)\left(\rho_{2}+\rho_{3}\right), \rho_{1} \rho_{2}\left(\rho_{2}+\rho_{3}\right), \rho_{1}\left(\rho_{1}+\rho_{2}\right)\left(\rho_{2}+\rho_{3}\right)\right.$, \\
& $\left.\rho_{3}\left(\rho_{1}+\rho_{3}\right)\left(\rho_{2}+\rho_{3}\right), \rho_{1} \rho_{3}\left(\rho_{2}+\rho_{3}\right), \rho_{1}\left(\rho_{1}+\rho_{3}\right)\left(\rho_{2}+\rho_{3}\right)\right\}$ \\
\hline
\end{tabular}

Then the lemma follows from the argument above and Corollary 2.2.

Remark 6.1. Lemma 6.4 also gives the method for constructing 42 different small covers (up to equivariant cobordism) with 6 fixed points. In particular, we easily see the property that for each $\left(S^{1} \times \mathbb{R} P^{2}, \Phi_{j}\right)$, two elements of $\mathcal{N}_{\left(S^{1} \times \mathbb{R} P^{2}, \Phi_{j}\right)}$ are in some $\mathcal{N}_{\left(\mathbb{R} P^{3}, T_{i}\right)}$, and the remaining elements are just distributed in four different 
$\mathcal{N}_{\left(\mathbb{R} P^{3}, T_{i_{1}}\right)}, \mathcal{N}_{\left(\mathbb{R} P^{3}, T_{i_{2}}\right)}, \mathcal{N}_{\left(\mathbb{R} P^{3}, T_{i_{3}}\right)}, \mathcal{N}_{\left(\mathbb{R} P^{3}, T_{i_{4}}\right)}$ with $i_{v} \neq i$ and $v=1,2,3$, 4. In addition, we also see from the argument of Lemma 6.4 that

$$
\begin{aligned}
& \delta_{3}\left(\left[\left(S^{1} \times \mathbb{R} P^{2}, \Phi_{0_{1}}\right)\right]+\left[\left(S^{1} \times \mathbb{R} P^{2}, \Phi_{0_{6}}\right)\right]+\left[\left(\mathbb{R} P^{3}, T_{0}\right)\right]\right)=0, \\
& \delta_{3}\left(\left[\left(S^{1} \times \mathbb{R} P^{2}, \Phi_{0_{2}}\right)\right]+\left[\left(S^{1} \times \mathbb{R} P^{2}, \Phi_{0_{5}}\right)\right]+\left[\left(\mathbb{R} P^{3}, T_{0}\right)\right]\right)=0, \\
& \delta_{3}\left(\left[\left(S^{1} \times \mathbb{R} P^{2}, \Phi_{0_{3}}\right)\right]+\left[\left(S^{1} \times \mathbb{R} P^{2}, \Phi_{0_{4}}\right)\right]+\left[\left(\mathbb{R} P^{3}, T_{0}\right)\right]\right)=0,
\end{aligned}
$$

where $\delta_{3}$ is the monomorphism of Theorem 2.1. This means that actually we need only to consider the half of the 42 small covers $\left(S^{1} \times \mathbb{R} P^{2}, \Phi_{j}\right)$ for $j=$ $0,1, \ldots, 41$, because up to equivariant cobordism the union of any two of them is not one of the $\left(\mathbb{R} P^{3}, T_{i}\right)$ for $i=0,1, \ldots, 6$. With no loss we may assume that such 21 different small covers are just $\left(S^{1} \times \mathbb{R} P^{2}, \Phi_{j}\right)$ for $j=0,1, \ldots, 20$, with their tangent representation sets as stated in Table II.

Now let $\beta \in \mathfrak{M}_{3}$ be an essential generator. By Lemma 3.1, one has that $\left|\mathcal{N}_{\beta}\right| \leq 14$.

Claim 1. $\left|\mathcal{N}_{\beta}\right|$ must be less than 12.

Proof. If $\left|\mathcal{N}_{\beta}\right|=14$, then for each $i=0,1, \ldots, 6$, there must be two monomials $\delta_{1}^{(i)}$ and $\delta_{2}^{(i)}$ in $\mathcal{N}_{\left(\mathbb{R} P^{3}, T_{i}\right)}$ that are contained in $\mathcal{N}_{\beta}$. By Lemma 6.4 and Remark 6.1, an easy argument shows that there must be some $\left(S^{1} \times \mathbb{R} P^{2}, \Phi_{j}\right)$ such that $\mathcal{N}_{\left(S^{1} \times \mathbb{R} P^{2}, \Phi_{j}\right)} \subset \mathcal{N}_{\beta}$. Then $8=\left|\mathcal{N}_{\beta+\left[\left(S^{1} \times \mathbb{R} P^{2}, \Phi_{j}\right)\right]}\right|<\left|\mathcal{N}_{\beta}\right|=14$. However, this is a contradiction since $\beta$ is an essential generator. Thus $\left|\mathcal{N}_{\beta}\right|=14$ is impossible.

If $\left|\mathcal{N}_{\beta}\right|=12$, since each $\mathcal{N}_{\left(\mathbb{R} P^{3}, T_{i}\right)}$ contains at most two monomials in $\mathcal{N}_{\beta}$, the set $\mathcal{N}_{\beta}=\left\{\delta_{1}, \delta_{2}, \ldots, \delta_{11}, \delta_{12}\right\}$ splits into two possible cases: (i) $\mathcal{N}_{\beta}$ is the disjoint union of the subsets $\left\{\delta_{1}, \delta_{2}\right\}, \ldots,\left\{\delta_{11}, \delta_{12}\right\}$ that are distributed in six different $\mathcal{N}_{\left(\mathbb{R} P^{3}, T_{i}\right)}$ or (ii) $\mathcal{N}_{\beta}$ is the disjoint union of seven subsets $\left\{\delta_{1}, \delta_{2}\right\}, \ldots,\left\{\delta_{9}, \delta_{10}\right\},\left\{\delta_{11}\right\},\left\{\delta_{12}\right\}$ that are just distributed in $\mathcal{N}_{\left(\mathbb{R} P^{3}, T_{0}\right)}, \ldots, \mathcal{N}_{\left(\mathbb{R} P^{3}, T_{6}\right)}$, respectively. A similar argument also shows that there must be some $\left(S^{1} \times \mathbb{R} P^{2}, \Phi_{j}\right)$ such that for the case (i), at least five elements of $\mathcal{N}_{\left(S^{1} \times \mathbb{R} P^{2}, \Phi_{j}\right)}$ are contained in $\mathcal{N}_{\beta}$, and for the case (ii), at least four elements of $\mathcal{N}_{\left(S^{1} \times \mathbb{R} P^{2}, \Phi_{j}\right)}$ are contained in $\mathcal{N}_{\beta}$. Then $\left|\mathcal{N}_{\beta+\left[\left(S^{1} \times \mathbb{R} P^{2}, \Phi_{j}\right)\right]}\right| \leq$ $10<\left|\mathcal{N}_{\beta}\right|=12$. This contradicts the fact that $\beta$ is an essential generator. Thus $\left|\mathcal{N}_{\beta}\right|=12$ cannot occur.

Let $(M, \phi)$ be a representative of $\beta$ such that $\mathcal{N}_{(M, \phi)}$ is prime, and let $\left(\Gamma_{(M, \phi)}, \alpha\right)$ be the moment graph of $(M, \phi)$.

Claim 2. $\Gamma_{(M, \phi)}$ is connected.

Proof. Suppose the contrary. Let $\Gamma^{\prime}$ be a connected component of $\Gamma_{(M, \phi)}$. Then the restriction $\left.\alpha\right|_{\Gamma^{\prime}}$ is still an axial function of $\Gamma^{\prime}$. By Claim 1, one has $\left|\mathcal{N}_{(M, \phi)}\right| \leq 10$, so the number of vertices of $\Gamma_{M}$ is at most 10 . If $\left|V_{\Gamma^{\prime}}\right|=2$, then obviously $\alpha\left(E_{p_{1}}\right)=\alpha\left(E_{p_{2}}\right)$ for $p_{1}, p_{2} \in V_{\Gamma^{\prime}}$, but this is impossible since $\mathcal{N}_{(M, \phi)}$ is prime. If $\left|V_{\Gamma^{\prime}}\right|=4$, then $\Gamma^{\prime}$ must be the 1-skeleton of a 3 -simplex, and thus $\left(\Gamma^{\prime},\left.\alpha\right|_{\Gamma^{\prime}}\right)$ is 


\begin{tabular}{|c|c|}
\hline$\left(S^{1} \times \mathbb{R} P^{2}, \Phi_{0}\right)$ & $\begin{array}{l}\rho_{1} \rho_{2} \rho_{3}, \rho_{1} \rho_{2}\left(\rho_{2}+\rho_{3}\right), \rho_{1} \rho_{3}\left(\rho_{2}+\rho_{3}\right), \rho_{1}\left(\rho_{1}+\rho_{2}\right)\left(\rho_{1}+\rho_{3}\right), \\
\rho_{1}\left(\rho_{1}+\rho_{2}\right)\left(\rho_{2}+\rho_{3}\right), \rho_{1}\left(\rho_{1}+\rho_{3}\right)\left(\rho_{2}+\rho_{3}\right)\end{array}$ \\
\hline$\left(S^{1} \times \mathbb{R} P^{2}, \Phi_{1}\right)$ & $\begin{array}{l}\rho_{1} \rho_{2} \rho_{3}, \rho_{1} \rho_{2}\left(\rho_{1}+\rho_{3}\right), \rho_{2} \rho_{3}\left(\rho_{1}+\rho_{3}\right), \rho_{2}\left(\rho_{1}+\rho_{2}\right)\left(\rho_{1}+\rho_{3}\right), \\
\rho_{2}\left(\rho_{1}+\rho_{2}\right)\left(\rho_{2}+\rho_{3}\right), \rho_{2}\left(\rho_{1}+\rho_{3}\right)\left(\rho_{2}+\rho_{3}\right)\end{array}$ \\
\hline$\left(S^{1} \times \mathbb{R} P^{2}, \Phi_{2}\right)$ & $\begin{array}{l}\rho_{1} \rho_{2} \rho_{3}, \rho_{1} \rho_{3}\left(\rho_{1}+\rho_{2}\right), \rho_{2} \rho_{3}\left(\rho_{1}+\rho_{2}\right), \rho_{3}\left(\rho_{1}+\rho_{2}\right)\left(\rho_{1}+\rho_{3}\right), \\
\rho_{3}\left(\rho_{1}+\rho_{2}\right)\left(\rho_{2}+\rho_{3}\right), \rho_{3}\left(\rho_{1}+\rho_{3}\right)\left(\rho_{2}+\rho_{3}\right)\end{array}$ \\
\hline$\left(S^{1} \times \mathbb{R} P^{2}, \Phi_{3}\right)$ & $\begin{array}{l}\rho_{1} \rho_{2} \rho_{3}, \rho_{1} \rho_{2}\left(\rho_{1}+\rho_{3}\right), \rho_{2} \rho_{3}\left(\rho_{1}+\rho_{2}\right), \rho_{2} \rho_{3}\left(\rho_{1}+\rho_{3}\right) \\
\rho_{2} \rho_{3}\left(\rho_{1}+\rho_{2}+\rho_{3}\right), \rho_{2}\left(\rho_{1}+\rho_{2}\right)\left(\rho_{1}+\rho_{2}+\rho_{3}\right)\end{array}$ \\
\hline$\left(S^{1} \times \mathbb{R} P^{2}, \Phi_{4}\right)$ & $\begin{array}{l}\rho_{1} \rho_{2}\left(\rho_{1}+\rho_{2}+\rho_{3}\right), \rho_{1} \rho_{3}\left(\rho_{1}+\rho_{2}+\rho_{3}\right), \\
\rho_{1}\left(\rho_{1}+\rho_{2}\right)\left(\rho_{1}+\rho_{2}+\rho_{3}\right), \rho_{1}\left(\rho_{1}+\rho_{3}\right)\left(\rho_{1}+\rho_{2}+\rho_{3}\right), \\
\rho_{2}\left(\rho_{1}+\rho_{2}\right)\left(\rho_{1}+\rho_{2}+\rho_{3}\right), \rho_{3}\left(\rho_{1}+\rho_{3}\right)\left(\rho_{1}+\rho_{2}+\rho_{3}\right)\end{array}$ \\
\hline$\left(S^{1} \times \mathbb{R} P^{2}, \Phi_{5}\right)$ & $\begin{array}{l}\rho_{1} \rho_{3}\left(\rho_{1}+\rho_{2}\right), \rho_{1}\left(\rho_{1}+\rho_{2}\right)\left(\rho_{1}+\rho_{3}\right) \\
\rho_{2}\left(\rho_{1}+\rho_{2}\right)\left(\rho_{1}+\rho_{3}\right), \rho_{3}\left(\rho_{1}+\rho_{2}\right)\left(\rho_{1}+\rho_{3}\right) \\
\rho_{2}\left(\rho_{1}+\rho_{2}\right)\left(\rho_{1}+\rho_{2}+\rho_{3}\right),\left(\rho_{1}+\rho_{2}\right)\left(\rho_{1}+\rho_{3}\right)\left(\rho_{1}+\rho_{2}+\rho_{3}\right)\end{array}$ \\
\hline$\left(S^{1} \times \mathbb{R} P^{2}, \Phi_{6}\right)$ & $\begin{array}{l}\rho_{1} \rho_{2} \rho_{3}, \rho_{1} \rho_{2}\left(\rho_{2}+\rho_{3}\right), \rho_{1} \rho_{3}\left(\rho_{1}+\rho_{2}\right), \rho_{1} \rho_{3}\left(\rho_{2}+\rho_{3}\right) \\
\rho_{1} \rho_{3}\left(\rho_{1}+\rho_{2}+\rho_{3}\right), \rho_{1}\left(\rho_{1}+\rho_{2}\right)\left(\rho_{1}+\rho_{2}+\rho_{3}\right)\end{array}$ \\
\hline$\left(S^{1} \times \mathbb{R} P^{2}, \Phi_{7}\right)$ & $\begin{array}{l}\rho_{1} \rho_{2}\left(\rho_{1}+\rho_{2}+\rho_{3}\right), \rho_{2} \rho_{3}\left(\rho_{1}+\rho_{2}+\rho_{3}\right) \\
\rho_{1}\left(\rho_{1}+\rho_{2}\right)\left(\rho_{1}+\rho_{2}+\rho_{3}\right), \rho_{2}\left(\rho_{1}+\rho_{2}\right)\left(\rho_{1}+\rho_{2}+\rho_{3}\right) \\
\rho_{2}\left(\rho_{2}+\rho_{3}\right)\left(\rho_{1}+\rho_{2}+\rho_{3}\right), \rho_{3}\left(\rho_{2}+\rho_{3}\right)\left(\rho_{1}+\rho_{2}+\rho_{3}\right)\end{array}$ \\
\hline$\left(S^{1} \times \mathbb{R} P^{2}, \Phi_{8}\right)$ & $\begin{array}{l}\rho_{2} \rho_{3}\left(\rho_{1}+\rho_{2}\right), \rho_{2}\left(\rho_{1}+\rho_{2}\right)\left(\rho_{2}+\rho_{3}\right), \rho_{1}\left(\rho_{1}+\rho_{2}\right)\left(\rho_{2}+\rho_{3}\right) \\
\rho_{3}\left(\rho_{1}+\rho_{2}\right)\left(\rho_{2}+\rho_{3}\right), \rho_{1}\left(\rho_{1}+\rho_{2}\right)\left(\rho_{1}+\rho_{2}+\rho_{3}\right) \\
\left(\rho_{1}+\rho_{2}\right)\left(\rho_{2}+\rho_{3}\right)\left(\rho_{1}+\rho_{2}+\rho_{3}\right)\end{array}$ \\
\hline$\left(S^{1} \times \mathbb{R} P^{2}, \Phi_{9}\right)$ & $\begin{array}{l}\rho_{1} \rho_{2} \rho_{3}, \rho_{1} \rho_{2}\left(\rho_{1}+\rho_{3}\right), \rho_{1} \rho_{2}\left(\rho_{2}+\rho_{3}\right), \\
\rho_{1} \rho_{2}\left(\rho_{1}+\rho_{2}+\rho_{3}\right), \rho_{1} \rho_{3}\left(\rho_{2}+\rho_{3}\right), \rho_{1}\left(\rho_{1}+\rho_{3}\right)\left(\rho_{1}+\rho_{2}+\rho_{3}\right)\end{array}$ \\
\hline$\left(S^{1} \times \mathbb{R} P^{2}, \Phi_{10}\right)$ & $\begin{array}{l}\rho_{1} \rho_{3}\left(\rho_{1}+\rho_{2}+\rho_{3}\right), \rho_{2} \rho_{3}\left(\rho_{1}+\rho_{2}+\rho_{3}\right) \\
\rho_{1}\left(\rho_{1}+\rho_{3}\right)\left(\rho_{1}+\rho_{2}+\rho_{3}\right), \rho_{2}\left(\rho_{2}+\rho_{3}\right)\left(\rho_{1}+\rho_{2}+\rho_{3}\right) \\
\rho_{3}\left(\rho_{1}+\rho_{3}\right)\left(\rho_{1}+\rho_{2}+\rho_{3}\right), \rho_{3}\left(\rho_{2}+\rho_{3}\right)\left(\rho_{1}+\rho_{2}+\rho_{3}\right)\end{array}$ \\
\hline$\left(S^{1} \times \mathbb{R} P^{2}, \Phi_{11}\right)$ & $\begin{array}{l}\rho_{2} \rho_{3}\left(\rho_{1}+\rho_{3}\right), \rho_{1}\left(\rho_{1}+\rho_{3}\right)\left(\rho_{2}+\rho_{3}\right), \\
\rho_{1}\left(\rho_{1}+\rho_{3}\right)\left(\rho_{1}+\rho_{2}+\rho_{3}\right), \rho_{2}\left(\rho_{1}+\rho_{3}\right)\left(\rho_{2}+\rho_{3}\right), \\
\rho_{3}\left(\rho_{1}+\rho_{3}\right)\left(\rho_{2}+\rho_{3}\right),\left(\rho_{1}+\rho_{3}\right)\left(\rho_{2}+\rho_{3}\right)\left(\rho_{1}+\rho_{2}+\rho_{3}\right)\end{array}$ \\
\hline
\end{tabular}

Table II

the moment graph of some $\left(\mathbb{R} P^{3}, T_{i}\right)$. Further, the disjoint union of $(M, \phi)$ and $\left(\mathbb{R} P^{3}, T_{i}\right)$ forms a $\left(\mathbb{Z}_{2}\right)^{3}$-action with at most six fixed points. This contradicts the assumption that $\beta$ is an essential generator. If $\left|V_{\Gamma^{\prime}}\right|=6$, then, since the number of vertices of $\Gamma_{(M, \phi)}$ is at most $10, \Gamma_{(M, \phi)}$ must have another connected component 


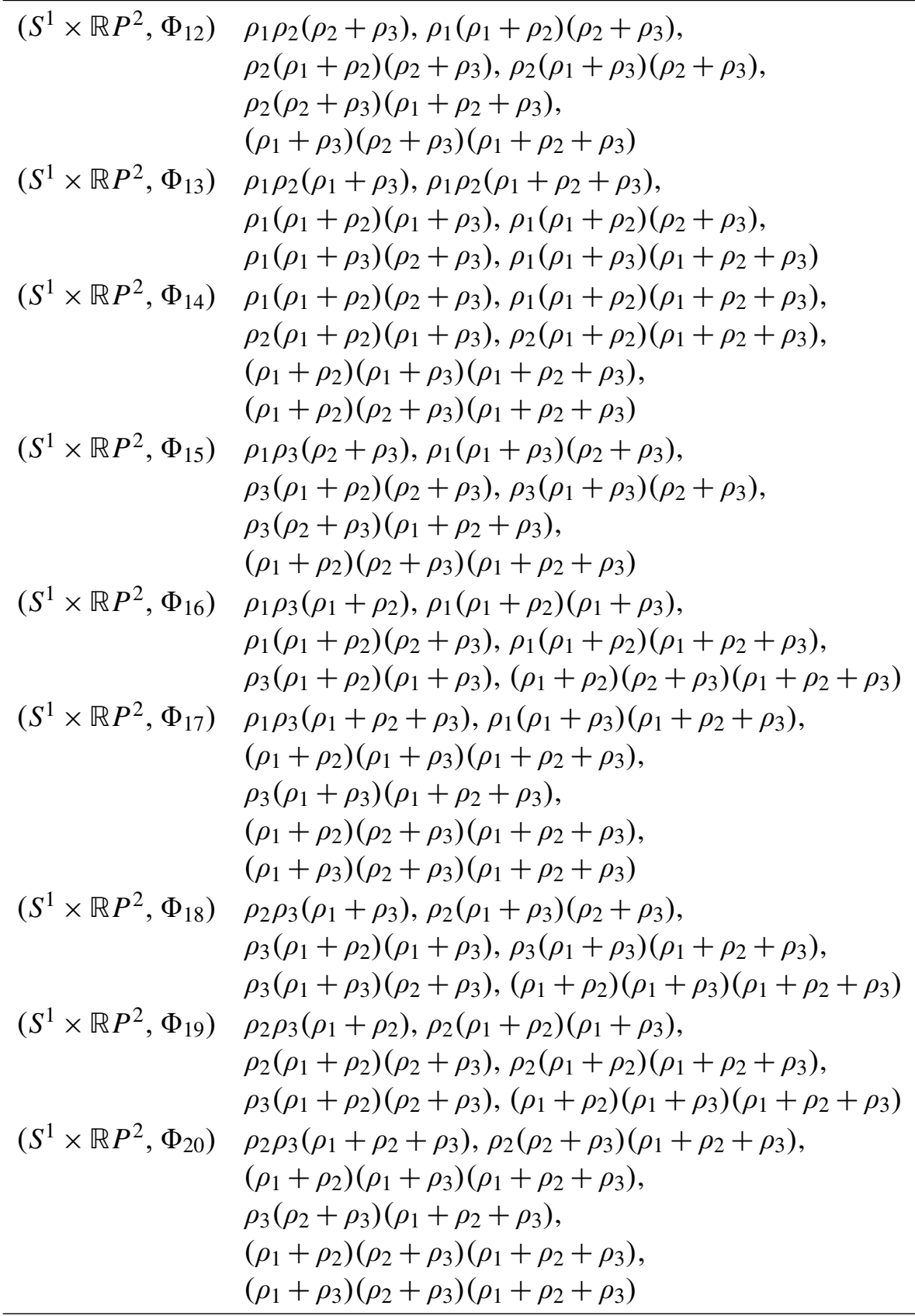

Table II continued.

with 2 or 4 vertices. This reduces the problem to the case $\left|V_{\Gamma^{\prime}}\right|=2$ or 4 and completes the proof. 
By Proposition 5.1 and Claim 2, the 2-skeletal expansion $N$ of $\left(\Gamma_{(M, \phi)}, \alpha\right)$ is a connected closed surface. Write $F_{\Gamma_{(M, \phi)}}$ for the set of all 2-nests in $\mathscr{K}_{\left(\Gamma_{(M, \phi)}, \alpha\right)}$. Then one has the formula

$$
\chi(N)=\left|V_{\Gamma_{(M, \phi)}}\right|-\left|E_{\Gamma_{(M, \phi)}}\right|+\left|F_{\Gamma_{(M, \phi)}}\right|,
$$

where $\chi(N)$ is the Euler characteristic of $N$. Note that $\left|V_{\Gamma_{(M, \phi)}}\right|=\left|\mathcal{N}_{\beta}\right|$ and $3\left|V_{\Gamma_{(M, \phi)}}\right|=2\left|E_{\Gamma_{(M, \phi)}}\right|$.

Claim 3. The 2-skeletal expansion $N$ of $\left(\Gamma_{(M, \phi)}, \alpha\right)$ is a sphere of dimension 2.

Proof. It suffices to show that the Euler characteristic $\chi(N)$ is 2 . By Claim 1, one has $\left|\mathcal{N}_{(M, \phi)}\right| \leq 10$, so one needs to consider the cases $\left|\mathcal{N}_{(M, \phi)}\right|=4,6,8,10$.

First, the case $\left|\mathcal{N}_{(M, \phi)}\right|=4$. If $\chi(N)$ is not 2, we have from (6-1) $\left|F_{\Gamma_{(M, \phi)}}\right| \leq 3$, so all 2-nests in $\left(\Gamma_{(M, \phi)}, \alpha\right)$ correspond to no more than three nonzero elements in $\operatorname{Hom}\left(\mathbb{Z}_{2},\left(\mathbb{Z}_{2}\right)^{3}\right)$. However, any three nonzero elements therein cannot produce four different bases therein. Thus, $\chi(N)$ must be 2 .

Next, the case $\left|\mathcal{N}_{(M, \phi)}\right|=6$. Since any four nonzero elements in $\operatorname{Hom}\left(\mathbb{Z}_{2},\left(\mathbb{Z}_{2}\right)^{3}\right)$ cannot produce six different bases there, $\left|F_{\Gamma_{(M, \phi)}}\right|$ must be 5 and so $\chi(N)$ is 2 .

The case $\left|\mathcal{N}_{(M, \phi)}\right|=8$. If $N$ is not a sphere of dimension 2, then the argument above insures that $\left|F_{\Gamma_{(M, \phi)}}\right|$ must be 5, and the dual map $\eta$ of $\alpha$ maps five 2-nests of $\mathscr{K}_{\left(\Gamma_{(M, \phi)}, \alpha\right)}$ to five different nonzero elements of $\operatorname{Hom}\left(\mathbb{Z}_{2},\left(\mathbb{Z}_{2}\right)^{3}\right)$. An easy argument shows that any five such nonzero elements can be translated into five given nonzero elements by applying an automorphism of $\operatorname{Hom}\left(\mathbb{Z}_{2},\left(\mathbb{Z}_{2}\right)^{3}\right)$. Thus we may choose five special elements $\rho_{1}^{*}, \rho_{2}^{*}, \rho_{3}^{*}, \rho_{1}^{*}+\rho_{2}^{*}, \rho_{1}^{*}+\rho_{3}^{*}$ as being the images of $\eta$ on five 2-nests of $\mathscr{K}_{\left(\Gamma_{(M, \phi)}, \alpha\right)}$, where $\left\{\rho_{1}^{*}, \rho_{2}^{*}, \rho_{3}^{*}\right\}$ is the standard basis of $\operatorname{Hom}\left(\mathbb{Z}_{2},\left(\mathbb{Z}_{2}\right)^{3}\right)$, dual to the standard basis $\left\{\rho_{1}, \rho_{2}, \rho_{3}\right\}$ of $\operatorname{Hom}\left(\left(\mathbb{Z}_{2}\right)^{3}, \mathbb{Z}_{2}\right)$. Then from these five chosen elements, one may produce just 8 bases of $\operatorname{Hom}\left(\mathbb{Z}_{2},\left(\mathbb{Z}_{2}\right)^{3}\right)$, given by

$$
\begin{gathered}
\left\{\rho_{1}^{*}, \rho_{2}^{*}, \rho_{3}^{*}\right\},\left\{\rho_{1}^{*}, \rho_{2}^{*}, \rho_{1}^{*}+\rho_{3}^{*}\right\},\left\{\rho_{1}^{*}, \rho_{3}^{*}, \rho_{1}^{*}+\rho_{2}^{*}\right\},\left\{\rho_{1}^{*}, \rho_{1}^{*}+\rho_{2}^{*}, \rho_{1}^{*}+\rho_{3}^{*}\right\}, \\
\left\{\rho_{2}^{*}, \rho_{3}^{*}, \rho_{1}^{*}+\rho_{2}^{*}\right\},\left\{\rho_{2}^{*}, \rho_{3}^{*}, \rho_{1}^{*}+\rho_{3}^{*}\right\},\left\{\rho_{2}^{*}, \rho_{1}^{*}+\rho_{2}^{*}, \rho_{1}^{*}+\rho_{3}^{*}\right\},\left\{\rho_{3}^{*}, \rho_{1}^{*}+\rho_{2}^{*}, \rho_{1}^{*}+\rho_{3}^{*}\right\} .
\end{gathered}
$$

So, $\mathcal{N}_{(M, \phi)}$ consists of 8 monomials

$$
\rho_{1} \rho_{2} \rho_{3}, \rho_{2} \rho_{3}\left(\rho_{1}+\rho_{3}\right), \rho_{2} \rho_{3}\left(\rho_{1}+\rho_{2}\right), \rho_{2} \rho_{3}\left(\rho_{1}+\rho_{2}+\rho_{3}\right),
$$

$\rho_{1} \rho_{3}\left(\rho_{1}+\rho_{2}\right), \rho_{1} \rho_{2}\left(\rho_{1}+\rho_{3}\right), \rho_{3}\left(\rho_{1}+\rho_{3}\right)\left(\rho_{1}+\rho_{2}+\rho_{3}\right), \rho_{2}\left(\rho_{1}+\rho_{2}\right)\left(\rho_{1}+\rho_{2}+\rho_{3}\right)$.

Further, we see from Table I that $\mathcal{N}_{\left(\mathbb{R} P^{3}, T_{3}\right)} \subset \mathcal{N}_{(M, \phi)}$, so $\left|\mathcal{N}_{\beta+\left[\left(\mathbb{R} P^{3}, T_{3}\right)\right]}\right|<8$. This means that $\beta$ is not an essential generator, which gives a contradiction. Thus $\left|F_{\Gamma_{(M, \phi)}}\right|$ must be 6 when $\left|\mathcal{N}_{(M, \phi)}\right|=8$, and so $\chi(N)$ is still 2 .

When $\left|\mathcal{N}_{(M, \phi)}\right|=10$, suppose that $\chi(N)$ is not 2. As shown above, any five nonzero elements in $\operatorname{Hom}\left(\mathbb{Z}_{2},\left(\mathbb{Z}_{2}\right)^{3}\right)$ cannot produce ten different bases in it, and thus the only possibility of $\left|F_{\Gamma_{(M, \phi)}}\right|$ is 6 . Further, one has from (6-1) that $\chi(N)$ must be 1. To ensure that $\left|\mathcal{N}_{(M, \phi)}\right|=10$, six 2-nests in $\mathcal{K}_{\left(\Gamma_{(M, \phi)}, \alpha\right)}$ must then correspond 
via the dual map $\eta$ to six different nonzero elements in $\operatorname{Hom}\left(\mathbb{Z}_{2},\left(\mathbb{Z}_{2}\right)^{3}\right)$. It is easy to check that any six different nonzero elements there can still be translated into the given six different nonzero elements by an automorphism. Thus, as in the argument of the case $\left|\mathcal{N}_{(M, \phi)}\right|=8$, one needs to merely consider six special nonzero elements of $\operatorname{Hom}\left(\mathbb{Z}_{2},\left(\mathbb{Z}_{2}\right)^{3}\right)$. Take six nonzero elements $\rho_{1}^{*}, \rho_{2}^{*}, \rho_{3}^{*}, \rho_{1}^{*}+\rho_{2}^{*}, \rho_{1}^{*}+\rho_{3}^{*}$ and $\rho_{1}^{*}+\rho_{2}^{*}+\rho_{3}^{*}$ that are the images by $\eta$ on six 2-nests. One then may produce 16 different bases of $\operatorname{Hom}\left(\mathbb{Z}_{2},\left(\mathbb{Z}_{2}\right)^{3}\right)$, given by

$\left\{\rho_{1}^{*}, \rho_{2}^{*}, \rho_{3}^{*}\right\},\left\{\rho_{2}^{*}, \rho_{3}^{*}, \rho_{1}^{*}+\rho_{2}^{*}+\rho_{3}^{*}\right\},\left\{\rho_{1}^{*}, \rho_{3}^{*}, \rho_{1}^{*}+\rho_{2}^{*}+\rho_{3}^{*}\right\},\left\{\rho_{1}^{*}, \rho_{2}^{*}, \rho_{1}^{*}+\rho_{2}^{*}+\rho_{3}^{*}\right\}$, $\left\{\rho_{3}^{*}, \rho_{1}^{*}+\rho_{2}^{*}, \rho_{1}^{*}+\rho_{3}^{*}\right\},\left\{\rho_{2}^{*}, \rho_{3}^{*}, \rho_{1}^{*}+\rho_{3}^{*}\right\},\left\{\rho_{2}^{*}, \rho_{1}^{*}+\rho_{2}^{*}, \rho_{1}^{*}+\rho_{3}^{*}\right\}$, $\left\{\rho_{2}^{*}, \rho_{3}^{*}, \rho_{1}^{*}+\rho_{2}^{*}\right\},\left\{\rho_{1}^{*}+\rho_{2}^{*}, \rho_{1}^{*}+\rho_{3}^{*}, \rho_{1}^{*}+\rho_{2}^{*}+\rho_{3}^{*}\right\},\left\{\rho_{1}^{*}, \rho_{1}^{*}+\rho_{3}^{*}, \rho_{1}^{*}+\rho_{2}^{*}+\rho_{3}^{*}\right\}$, $\left\{\rho_{1}^{*}, \rho_{1}^{*}+\rho_{2}^{*}, \rho_{1}^{*}+\rho_{2}^{*}+\rho_{3}^{*}\right\},\left\{\rho_{1}^{*}, \rho_{1}^{*}+\rho_{2}^{*}, \rho_{1}^{*}+\rho_{3}^{*}\right\},\left\{\rho_{1}^{*}, \rho_{3}^{*}, \rho_{1}^{*}+\rho_{2}^{*}\right\}$, $\left\{\rho_{1}^{*}, \rho_{2}^{*}, \rho_{1}^{*}+\rho_{3}^{*}\right\},\left\{\rho_{3}^{*}, \rho_{1}^{*}+\rho_{3}^{*}, \rho_{1}^{*}+\rho_{2}^{*}+\rho_{3}^{*}\right\},\left\{\rho_{2}^{*}, \rho_{1}^{*}+\rho_{2}^{*}, \rho_{1}^{*}+\rho_{2}^{*}+\rho_{3}^{*}\right\}$.

These 16 bases are dual to these 16 bases in $\operatorname{Hom}\left(\left(\mathbb{Z}_{2}\right)^{3}, \mathbb{Z}_{2}\right)$ :

$\rho_{1} \rho_{2} \rho_{3}, \rho_{1}\left(\rho_{1}+\rho_{2}\right)\left(\rho_{1}+\rho_{3}\right), \rho_{2}\left(\rho_{1}+\rho_{2}\right)\left(\rho_{2}+\rho_{3}\right), \rho_{1} \rho_{2}\left(\rho_{1}+\rho_{2}+\rho_{3}\right)$, $\rho_{2}\left(\rho_{1}+\rho_{2}\right)\left(\rho_{1}+\rho_{2}+\rho_{3}\right), \rho_{1} \rho_{2}\left(\rho_{1}+\rho_{3}\right), \rho_{3}\left(\rho_{1}+\rho_{3}\right)\left(\rho_{1}+\rho_{2}+\rho_{3}\right), \rho_{1} \rho_{3}\left(\rho_{1}+\rho_{2}\right)$, $\left(\rho_{1}+\rho_{2}\right)\left(\rho_{1}+\rho_{3}\right)\left(\rho_{1}+\rho_{2}+\rho_{3}\right), \rho_{2}\left(\rho_{1}+\rho_{3}\right)\left(\rho_{2}+\rho_{3}\right), \rho_{3}\left(\rho_{1}+\rho_{2}\right)\left(\rho_{2}+\rho_{3}\right)$,

$$
\rho_{2} \rho_{3}\left(\rho_{1}+\rho_{2}+\rho_{3}\right) \text {, }
$$

$\rho_{2} \rho_{3}\left(\rho_{1}+\rho_{2}\right), \rho_{2} \rho_{3}\left(\rho_{1}+\rho_{3}\right), \rho_{2}\left(\rho_{1}+\rho_{2}\right)\left(\rho_{1}+\rho_{3}\right), \rho_{3}\left(\rho_{1}+\rho_{2}\right)\left(\rho_{1}+\rho_{3}\right)$.

One sees that the first row above is just $\mathcal{N}_{\left(\mathbb{R} P^{3}, T_{0}\right)}$, the second row is $\mathcal{N}_{\left(\mathbb{R} P^{3}, T_{3}\right)}$, and the third row is $\mathcal{N}_{\left(\mathbb{R} P^{3}, T_{6}\right)}$, but $\rho_{2} \rho_{3}\left(\rho_{1}+\rho_{2}\right), \rho_{2} \rho_{3}\left(\rho_{1}+\rho_{3}\right), \rho_{2}\left(\rho_{1}+\rho_{2}\right)\left(\rho_{1}+\rho_{3}\right)$, $\rho_{3}\left(\rho_{1}+\rho_{2}\right)\left(\rho_{1}+\rho_{3}\right)$ belong to $\mathcal{N}_{\left(\mathbb{R} P^{3}, T_{1}\right)}, \mathcal{N}_{\left(\mathbb{R} P^{3}, T_{2}\right)}, \mathcal{N}_{\left(\mathbb{R} P^{3}, T_{4}\right)}, \mathcal{N}_{\left(\mathbb{R} P^{3}, T_{5}\right)}$, respectively. Then $\mathcal{N}_{(M, \phi)}$ must contain $\rho_{2} \rho_{3}\left(\rho_{1}+\rho_{2}\right), \rho_{2} \rho_{3}\left(\rho_{1}+\rho_{3}\right), \rho_{2}\left(\rho_{1}+\rho_{2}\right)\left(\rho_{1}+\rho_{3}\right)$, $\rho_{3}\left(\rho_{1}+\rho_{2}\right)\left(\rho_{1}+\rho_{3}\right)$, and $\left|\mathcal{N}_{(M, \phi)} \cap \mathcal{N}_{\left(\mathbb{R} P^{3}, T_{i}\right)}\right|=2$ for $i=0,3,6$.

Now choose any two $\gamma_{1}, \gamma_{2}$ of $\rho_{2} \rho_{3}\left(\rho_{1}+\rho_{2}\right), \rho_{2} \rho_{3}\left(\rho_{1}+\rho_{3}\right), \rho_{2}\left(\rho_{1}+\rho_{2}\right)\left(\rho_{1}+\rho_{3}\right)$, $\rho_{3}\left(\rho_{1}+\rho_{2}\right)\left(\rho_{1}+\rho_{3}\right)$. Then it is easy to show that there is always one $\left(S^{1} \times \mathbb{R} P^{2}, \Phi_{j}\right)$ such that $\mathcal{N}_{\left(S^{1} \times \mathbb{R} P^{2}, \Phi_{j}\right)}$ contains $\gamma_{1}, \gamma_{2}$. Without loss of generality, we may let $\gamma_{1}=\rho_{2} \rho_{3}\left(\rho_{1}+\rho_{2}\right)$ and $\gamma_{2}=\rho_{2} \rho_{3}\left(\rho_{1}+\rho_{3}\right)$. Then one has that

$$
\begin{aligned}
\mathcal{N}_{\left(S^{1} \times \mathbb{R} P^{2}, \Phi_{j}\right)}=\left\{\rho_{2} \rho_{3}\left(\rho_{1}+\rho_{2}\right), \rho_{2} \rho_{3}\left(\rho_{1}+\rho_{3}\right), \rho_{1} \rho_{2} \rho_{3}, \rho_{1} \rho_{2}\left(\rho_{1}+\rho_{3}\right),\right. \\
\left.\rho_{2} \rho_{3}\left(\rho_{1}+\rho_{2}+\rho_{3}\right), \rho_{2}\left(\rho_{1}+\rho_{2}\right)\left(\rho_{1}+\rho_{2}+\rho_{3}\right)\right\}
\end{aligned}
$$

with $\rho_{1} \rho_{2} \rho_{3} \in \mathcal{N}_{\left(\mathbb{R} P^{3}, T_{0}\right)}, \quad \rho_{1} \rho_{2}\left(\rho_{1}+\rho_{3}\right), \rho_{2}\left(\rho_{1}+\rho_{2}\right)\left(\rho_{1}+\rho_{2}+\rho_{3}\right) \in \mathcal{N}_{\left(\mathbb{R} P^{3}, T_{3}\right)}$, $\rho_{2} \rho_{3}\left(\rho_{1}+\rho_{2}+\rho_{3}\right) \in \mathcal{N}_{\left(\mathbb{R} P^{3}, T_{6}\right)}$. If $\mathcal{N}_{(M, \phi)}$ contains at least two of the monomials $\rho_{1} \rho_{2} \rho_{3}, \rho_{1} \rho_{2}\left(\rho_{1}+\rho_{3}\right), \rho_{2} \rho_{3}\left(\rho_{1}+\rho_{2}+\rho_{3}\right), \rho_{2}\left(\rho_{1}+\rho_{2}\right)\left(\rho_{1}+\rho_{2}+\rho_{3}\right)$, we get that

$$
\left|\mathcal{N}_{\beta+\left[\left(S^{1} \times \mathbb{R} P^{2}, \Phi_{j}\right)\right]}\right|<10,
$$




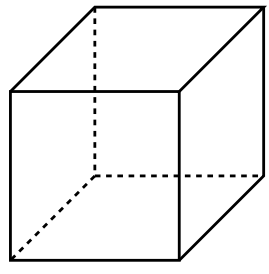

$P_{1}$

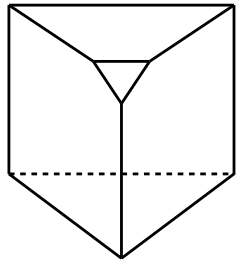

$P_{2}$

Figure 2. Two simple 3-polytopes with eight vertices.

which contradicts that $\beta$ is an essential generator. Thus, this case cannot occur. If $\mathcal{N}_{(M, \phi)}$ contains only one (say $\left.\omega\right)$ of $\rho_{1} \rho_{2} \rho_{3}, \rho_{1} \rho_{2}\left(\rho_{1}+\rho_{3}\right), \rho_{2} \rho_{3}\left(\rho_{1}+\rho_{2}+\rho_{3}\right)$, $\rho_{2}\left(\rho_{1}+\rho_{2}\right)\left(\rho_{1}+\rho_{2}+\rho_{3}\right)$, form the union $(M, \phi) \sqcup\left(S^{1} \times \mathbb{R} P^{2}, \Phi_{j}\right) \sqcup\left(\mathbb{R} P^{3}, T_{l}\right)$ where $l$ is chosen in $\{0,3,6\}$ so that $\omega \notin \mathcal{N}_{\left(\mathbb{R} P^{3}, T_{l}\right)}$. Then

$$
\left|\mathcal{N}_{\beta+\left[\left(S^{1} \times \mathbb{R} P^{2}, \Phi_{j}\right)\right]+\left[\left(\mathbb{R} P^{3}, T_{l}\right)\right]}\right|<10,
$$

which leads to a contradiction (note that $\left.\left|\mathcal{N}_{\left[\left(S^{1} \times \mathbb{R} P^{2}, \Phi_{j}\right)\right]+\left[\left(\mathbb{R} P^{3}, T_{l}\right)\right]}\right|<10\right)$. Finally, if $\mathcal{N}_{(M, \phi)}$ does not contain any one of $\rho_{1} \rho_{2} \rho_{3}, \rho_{1} \rho_{2}\left(\rho_{1}+\rho_{3}\right), \rho_{2} \rho_{3}\left(\rho_{1}+\rho_{2}+\rho_{3}\right)$, $\rho_{2}\left(\rho_{1}+\rho_{2}\right)\left(\rho_{1}+\rho_{2}+\rho_{3}\right)$, the disjoint union $(M, \phi) \sqcup\left(S^{1} \times \mathbb{R} P^{2}, \Phi_{j}\right) \sqcup\left(\mathbb{R} P^{3}, T_{3}\right)$ leads to the same type of contradiction. Therefore, $\chi(N)$ must be 2 .

Combining the above arguments, we complete the proof.

Lemma 6.5. Let $\beta \in \mathfrak{M}_{3}$. If $\beta$ is an essential generator, then $\left|\mathcal{N}_{\beta}\right| \leq 6$.

Proof. By Claim 1 it suffices to show that $\left|\mathcal{N}_{\beta}\right|$ is not equal to 8 and 10 . One knows by Claim 3 that the 2-skeletal expansion $N$ is a sphere of dimension 2, so $\Gamma_{(M, \phi)}$ is planar and, in particular, it is the 1-skeleton of a simple convex 3-polytope $P^{3}$. In this case, $M$ is a small cover over $P^{3}$, so the axial function $\alpha$ on $\Gamma_{(M, \phi)}$ is dual to the characteristic function $\lambda$ on $P^{3}$.

The argument proceeds as follows.

Case (i): $\left|\mathcal{N}_{\beta}\right|=8$. If $\left|\mathcal{N}_{\beta}\right|=8$, then $\Gamma_{(M, \phi)}$ is the 1-skeleton of a simple convex polytope with 8 vertices. From [Grünbaum 2003] one knows that there are only two different combinatorial types of simple 3-polytopes with eight vertices, as shown in Figure 2.

If $\Gamma_{(M, \phi)}$ is the 1-skeleton of a 3-dimensional cube $P_{1}$, then it is easy to check that $P_{1}$ does not admit any characteristic function that maps six 2-faces into six different nonzero elements in $\operatorname{Hom}\left(\mathbb{Z}_{2},\left(\mathbb{Z}_{2}\right)^{3}\right)$, but this is impossible. Thus, $\Gamma_{(M, \phi)}$ cannot be the 1-skeleton of $P_{1}$. If $\Gamma_{(M, \phi)}$ is the 1-skeleton of $P_{2}$, taking a triangular facet $F$ of $P_{2}$, then, up to automorphisms of $\operatorname{Hom}\left(\mathbb{Z}_{2},\left(\mathbb{Z}_{2}\right)^{3}\right)$, it is easy to see that the characteristic function $\lambda$ on $P_{2}$ maps $F$ with its 3 adjacent 2-faces into one of $\rho_{1}^{*}$, $\rho_{2}^{*}, \rho_{3}^{*}, \rho_{1}^{*}+\rho_{2}^{*}+\rho_{3}^{*}$ or $\rho_{1}^{*}, \rho_{2}^{*}, \rho_{3}^{*}, \rho_{1}^{*}+\rho_{2}^{*}$. In the first case, obviously there must 


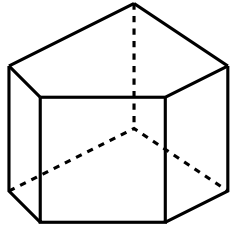

$P_{3}$

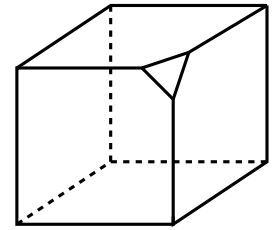

$P_{4}$

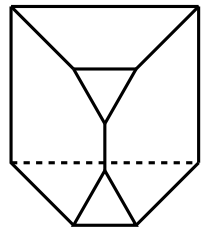

$P_{5}$
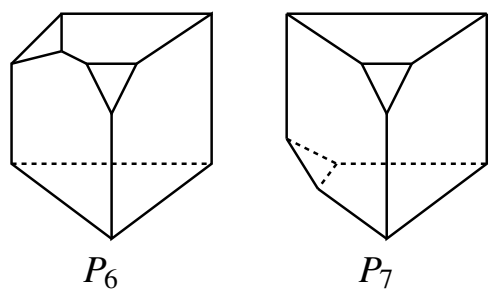

Figure 3. Simple 3-polytopes with ten vertices.

be some $\left(\mathbb{R} P^{3}, T_{i}\right)$ such that $\left|\mathcal{N}_{\beta+\left[\left(\mathbb{R} P^{3}, T_{i}\right)\right]}\right|=6<8$. This contradicts that $\beta$ is an essential generator, and thus this case cannot occur. In the second case, it is easy to check that there must be some $\left(S^{1} \times \mathbb{R} P^{2}, \Phi_{j}\right)$ such that $\left|\mathcal{N}_{\beta+\left[\left(S^{1} \times \mathbb{R} P^{2}, \Phi_{j}\right)\right]}\right|=6<8$. This also is impossible, so $\Gamma_{(M, \phi)}$ cannot be the 1-skeleton of $P_{2}$. Thus, if $\beta$ is an essential generator, $\left|\mathcal{N}_{\beta}\right|=8$ is impossible.

Case (ii): $\left|\mathcal{N}_{\beta}\right|=10$. If $\left|\mathcal{N}_{\beta}\right|=10$, then $\Gamma_{(M, \phi)}$ is the 1-skeleton of a simple convex polytope with 10 vertices. From [Grünbaum 2003] one knows that there are only five different combinatorial types of simple 3-polytopes with ten vertices, as shown in Figure 3. An easy argument shows that $\Gamma_{(M, \phi)}$ cannot be the 1-skeleton of $P_{3}$. Since each of $P_{4}, P_{5}, P_{6}, P_{7}$ has at least one triangular facet, similarly to the proof of Case (i), one may prove that $\Gamma_{(M, \phi)}$ cannot be the 1-skeleton of $P_{4}, P_{5}, P_{6}, P_{7}$. Therefore, $\left|\mathcal{N}_{\beta}\right|=10$ is impossible, which ends the proof.

Together with Lemmas 6.3 and 6.5 and Remark 6.1, we complete the proof of Proposition 6.2.

Theorem 6.6. As a vector space over $\mathbb{Z}_{2}, \mathfrak{M}_{3}$ has dimension 13 , and it is generated by

$$
\begin{gathered}
\left(\mathbb{R} P^{3}, T_{0}\right),\left(\mathbb{R} P^{3}, T_{1}\right), \ldots,\left(\mathbb{R} P^{3}, T_{6}\right), \\
\left(S^{1} \times \mathbb{R} P^{2}, \Phi_{0}\right),\left(S^{1} \times \mathbb{R} P^{2}, \Phi_{1}\right), \ldots,\left(S^{1} \times \mathbb{R} P^{2}, \Phi_{4}\right),\left(S^{1} \times \mathbb{R} P^{2}, \Phi_{6}\right) .
\end{gathered}
$$

Proof. By Propositions 3.2 and 6.2, any element of $\mathfrak{M}_{3}$ is a sum of elements of the set

$$
\left\{\left(\mathbb{R} P^{3}, T_{i}\right) \mid i=0,1, \ldots, 6\right\} \cup\left\{\left(S^{1} \times \mathbb{R} P^{2}, \Phi_{j}\right) \mid j=0,1, \ldots, 20\right\} .
$$


To calculate the dimension of $\mathfrak{M}_{3}$, one needs to determine a maximal linearly independent subset of this set. Let

$$
\left.\sum_{i=0}^{6} l_{i}\left[\left(\mathbb{R} P^{3}, T_{i}\right)\right]+\sum_{j=0}^{20} k_{j}\left[\left(S^{1} \times \mathbb{R} P^{2}\right), \Phi_{j}\right)\right]=0, \quad \text { where } l_{i}, k_{j} \in \mathbb{Z}_{2} .
$$

Using the Stong homomorphism $\delta_{3}$ in Theorem 2.1, one has that

$$
\left.\sum_{i=0}^{6} l_{i} \delta_{3}\left(\left[\left(\mathbb{R} P^{3}, T_{i}\right)\right]\right)+\sum_{j=0}^{20} k_{j} \delta_{3}\left(\left[\left(S^{1} \times \mathbb{R} P^{2}\right), \Phi_{j}\right)\right]\right)=0 .
$$

Since $\operatorname{Hom}\left(\left(\mathbb{Z}_{2}\right)^{3}, \mathbb{Z}_{2}\right)$ has 28 different bases, from (6-2) and Tables I and II, one obtains system of 28 linear equations, whose coefficient matrix $A$ is shown in Table III. By doing elementary row operations, $A$ is changed into the matrix $A^{\prime}$ whose first 13 rows are given in Table IV and whose last 15 rows are zero. Thus the rank of $A$ is 13 , which is just the dimension of $\mathfrak{M}_{3}$. Theorem 6.6 then follows from this.

\section{Representatives of equivariant cobordism classes of $\mathfrak{M}_{3}$}

Given two small covers $\pi_{i}: M_{i}^{n} \rightarrow P_{i}^{n}$ for $i=1,2$, their equivariant connected sum along fixed points can be proceeded as follows: Take a vertex $v_{i}$ of $P_{i}^{n}$ and let $p_{i}$ be its preimage in $M_{i}$ for $i=1,2$. With no loss one may assume that the underlying $\left(\mathbb{Z}_{2}\right)^{n}$-actions are equivalent in a neighborhood of $p_{i}$ (actually, if necessary, one can change the action by using an automorphism of $\left.\left(\mathbb{Z}_{2}\right)^{n}\right)$. Then one can perform the connected sum equivariantly near the fixed points $p_{1}$ and $p_{2}$. The result is a 2-torus manifold $M_{1}^{n} \sharp M_{2}^{n}$, and its orbit space $P_{1}^{n} \sharp P_{2}^{n}$ is given by removing a small ball around $v_{i}$ from $P_{i}^{n}$ and gluing the results together. As pointed out in [Davis and Januszkiewicz 1991], generally $P_{1}^{n} \sharp P_{2}^{n}$ is not canonically identified with a simple polytope but is almost as good in the sense that its boundary complex is dual to some PL triangulation of $S^{n-1}$. However, it is easy to see that if $n=3$, $P_{1}^{n} \sharp P_{2}^{n}$ is also a simple polytope, so $M_{1}^{n} \sharp M_{2}^{n}$ is a small cover over $P_{1}^{n} \sharp P_{2}^{n}$.

Lemma 7.1. There exists a 3-dimensional small cover $\pi: M^{3} \rightarrow P^{3}$ such that $M^{3}$ is equivariantly cobordant to a 2-torus 3-manifold $N^{3}$ with $\mathcal{N}_{N^{3}}$ prime and $\left|\mathcal{N}_{N^{3}}\right|=28$.

Proof. Consider the two small covers $\left(S^{1} \times \mathbb{R} P^{2}, \Phi_{0}\right)$ and $\left(S^{1} \times \mathbb{R} P^{2}, \Phi_{1}\right)$ and $P^{3}$ the corresponding prism. One sees from Table II that they have fixed points with the same representation $\rho_{1} \rho_{2} \rho_{3}$. Then one can make an equivariant connected sum along these fixed points so that $\left(S^{1} \times \mathbb{R} P^{2}, \Phi_{0}\right) \sharp\left(S^{1} \times \mathbb{R} P^{2}, \Phi_{1}\right)$ is also a small cover over a simple 3 -polytope with 10 vertices, and its tangent representation set 


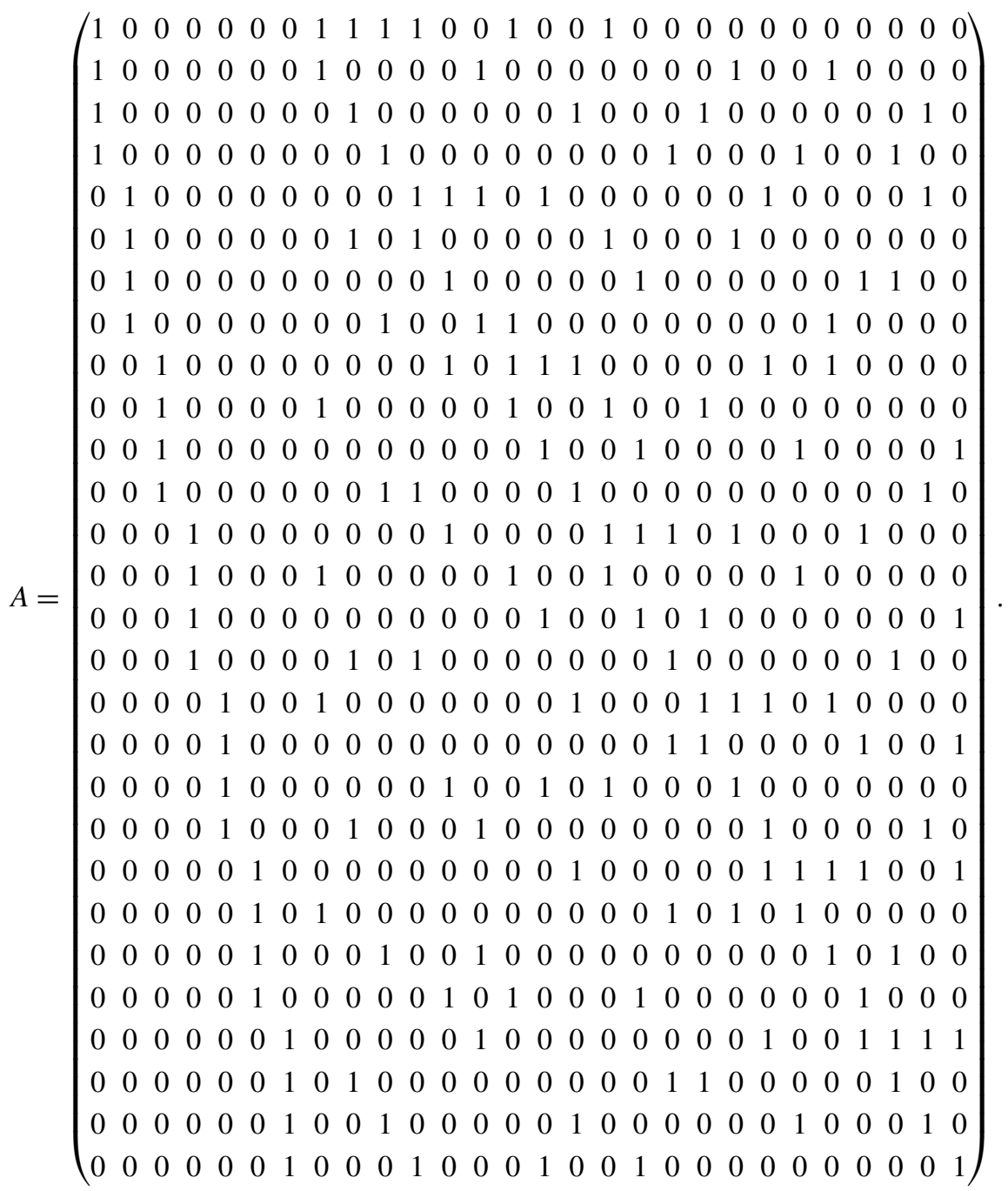

Table III. The coefficient matrix $A$.

is just equal to $\mathcal{N}_{\left[\left(S^{1} \times \mathbb{R} P^{2}, \Phi_{0}\right)\right]+\left[\left(S^{1} \times \mathbb{R} P^{2}, \Phi_{1}\right)\right]}$, consisting of

$$
\begin{gathered}
\rho_{1} \rho_{2}\left(\rho_{2}+\rho_{3}\right), \rho_{1} \rho_{3}\left(\rho_{2}+\rho_{3}\right), \rho_{1}\left(\rho_{1}+\rho_{2}\right)\left(\rho_{1}+\rho_{3}\right), \\
\rho_{1}\left(\rho_{1}+\rho_{2}\right)\left(\rho_{2}+\rho_{3}\right), \rho_{1}\left(\rho_{1}+\rho_{3}\right)\left(\rho_{2}+\rho_{3}\right), \rho_{1} \rho_{2}\left(\rho_{1}+\rho_{3}\right),
\end{gathered}
$$

$\rho_{2} \rho_{3}\left(\rho_{1}+\rho_{3}\right), \rho_{2}\left(\rho_{1}+\rho_{2}\right)\left(\rho_{1}+\rho_{3}\right), \rho_{2}\left(\rho_{1}+\rho_{2}\right)\left(\rho_{2}+\rho_{3}\right), \rho_{2}\left(\rho_{1}+\rho_{3}\right)\left(\rho_{2}+\rho_{3}\right)$.

From Table I one sees the following properties:

(a) $\mathcal{N}_{\left[\left(S^{1} \times \mathbb{R} P^{2}, \Phi_{0}\right)\right]+\left[\left(S^{1} \times \mathbb{R} P^{2}, \Phi_{1}\right)\right]}$ and $\mathcal{N}_{\left[\left(\mathbb{R} P^{3}, T_{i}\right)\right]}$ have nonempty intersection for any $\left(\mathbb{R} P^{3}, T_{i}\right)$. 


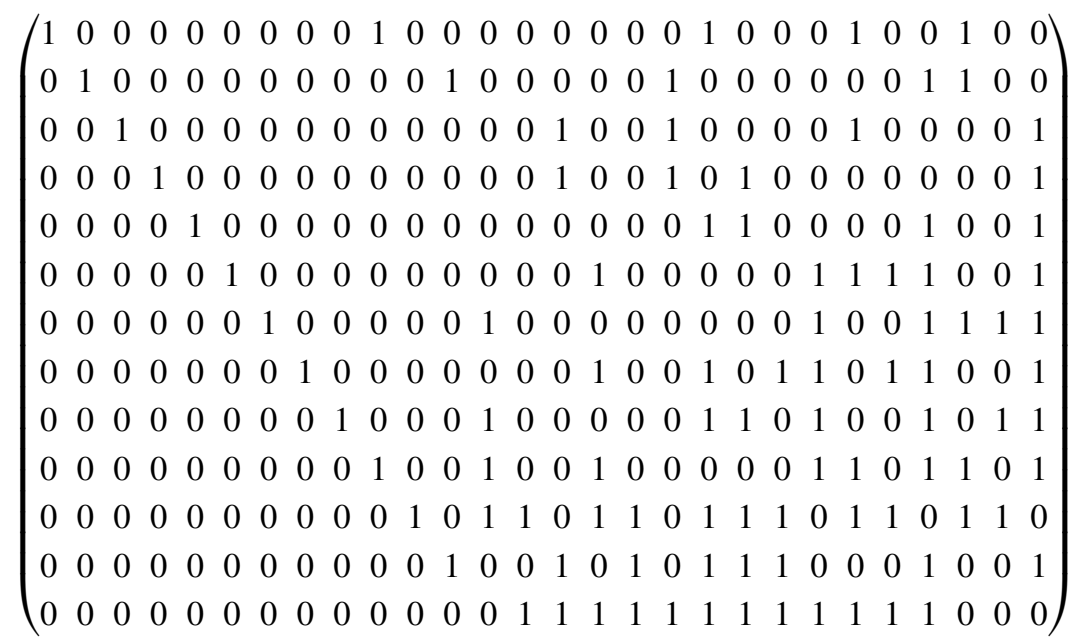

Table IV. The first 13 rows of the row reduction of $A$.

(b) $\mathcal{N}_{\left[\left(\mathbb{R} P^{3}, T_{0}\right)\right]}$ contains the elements $\rho_{1}\left(\rho_{1}+\rho_{2}\right)\left(\rho_{1}+\rho_{3}\right)$ and $\rho_{2}\left(\rho_{1}+\rho_{2}\right)\left(\rho_{2}+\rho_{3}\right)$ of $\mathcal{N}_{\left[\left(S^{1} \times \mathbb{R} P^{2}, \Phi_{0}\right)\right]+\left[\left(S^{1} \times \mathbb{R} P^{2}, \Phi_{1}\right)\right]}$

Next, one performs an equivariant connected sum of two copies of $\left(S^{1} \times \mathbb{R} P^{2}, \Phi_{0}\right) \sharp$ $\left(S^{1} \times \mathbb{R} P^{2}, \Phi_{1}\right)$ along the fixed point with representation $\rho_{1}\left(\rho_{1}+\rho_{2}\right)\left(\rho_{1}+\rho_{3}\right)$. Then the resulting $\left(\mathbb{Z}_{2}\right)^{3}$-manifold $M^{\prime}$ fixes 18 isolated points and is also a small cover over a simple polytope with 18 vertices. Obviously, the representations at the 18 fixed points of $M^{\prime}$ appear in pairs, so $M^{\prime}$ bounds equivariantly. Since $\mathcal{N}_{\left[\left(S^{1} \times \mathbb{R} P^{2}, \Phi_{0}\right)\right]+\left[\left(S^{1} \times \mathbb{R} P^{2}, \Phi_{1}\right)\right]} \backslash\left\{\rho_{1}\left(\rho_{1}+\rho_{2}\right)\left(\rho_{1}+\rho_{3}\right)\right\} \subset \mathcal{N}_{M^{\prime}}$, we have by properties (a) and (b) that for any $\left(\mathbb{R} P^{3}, T_{i}\right)$, the intersection $\mathcal{N}_{M^{\prime}} \cap \mathcal{N}_{\left[\left(\mathbb{R} P^{3}, T_{i}\right)\right]}$ is nonempty, and thus we can make an equivariant connected sum of $M^{\prime}$ with each $\left(\mathbb{R} P^{3}, T_{i}\right)$ along the fixed points with the same representation. Let $M$ be the equivariant connected sum of $M^{\prime}$ with all $\left(\mathbb{R} P^{3}, T_{i}\right)$ as above. Then $M$ is just the desired small cover. $\square$

Theorem 7.2. Any element $\beta$ in $\mathfrak{M}_{3}$ contains a small cover as its representative.

Proof. If $\beta=0$, then the bounding small cover $M^{\prime}$ of the proof of Lemma 7.1 can be chosen as a representative of $\beta$.

If $\beta \neq 0$, then $\beta$ is a sum of elements of the basis given in Theorem 6.6. Consider the small cover $M^{3}$ constructed in Lemma 7.1. Since $M^{3}$ is equivariantly cobordant to a 2-torus 3-manifold $N^{3}$ with $\mathcal{N}_{N^{3}}$ prime and $\left|\mathcal{N}_{N^{3}}\right|=28$, we can take a fixed point $p$ of $M^{3}$ with representation $\rho_{1} \rho_{2} \rho_{3}$. First one performs an equivariant connected sum $M^{3} \sharp M^{3}$ of two copies of $M^{3}$ along the fixed point $p$, so that $M^{3} \sharp M^{3}$ is also a small cover and bounds equivariantly. Thus, all tangent representations in $\mathcal{N}_{M^{3} \sharp M^{3}}$ appear in pairs. On the other hand, $\operatorname{Hom}\left(\left(\mathbb{Z}_{2}\right)^{3}, \mathbb{Z}_{2}\right)$ has 28 different bases, which gives 28 monomials of degree 3 in $\mathbb{Z}_{2}\left[\rho_{1}, \rho_{2}, \rho_{3}\right]$. Let $\mathscr{T}$ 
denote the set of these 28 monomials of degree 3 in $\mathbb{Z}_{2}\left[\rho_{1}, \rho_{2}, \rho_{3}\right]$. Then we have that $\mathcal{N}_{M^{3} \sharp M^{3}}$ must contain the 27 monomials of $\mathscr{T} \backslash\left\{\rho_{1} \rho_{2} \rho_{3}\right\}$. Next, we see from Tables I and II that we can choose a fixed point from each of the 13 small covers listed in Theorem 6.6 such that the tangent representations at those 13 chosen fixed points are not isomorphic to each other, and they are all in $\mathcal{N}_{M^{3} \sharp M^{3}}$. For example, we can choose the required tangent representations as

$$
\begin{gathered}
\rho_{1}\left(\rho_{1}+\rho_{2}\right)\left(\rho_{1}+\rho_{3}\right), \rho_{1} \rho_{2}\left(\rho_{2}+\rho_{3}\right), \rho_{1} \rho_{3}\left(\rho_{2}+\rho_{3}\right), \rho_{1} \rho_{3}\left(\rho_{1}+\rho_{2}\right), \\
\rho_{1} \rho_{2}\left(\rho_{1}+\rho_{2}+\rho_{3}\right), \rho_{1} \rho_{3}\left(\rho_{1}+\rho_{2}+\rho_{3}\right), \rho_{2} \rho_{3}\left(\rho_{1}+\rho_{2}+\rho_{3}\right), \\
\rho_{2}\left(\rho_{1}+\rho_{2}\right)\left(\rho_{1}+\rho_{3}\right), \rho_{3}\left(\rho_{1}+\rho_{2}\right)\left(\rho_{1}+\rho_{3}\right), \rho_{2}\left(\rho_{1}+\rho_{2}\right)\left(\rho_{1}+\rho_{2}+\rho_{3}\right), \\
\rho_{3}\left(\rho_{1}+\rho_{3}\right)\left(\rho_{1}+\rho_{2}+\rho_{3}\right),\left(\rho_{1}+\rho_{2}\right)\left(\rho_{1}+\rho_{3}\right)\left(\rho_{1}+\rho_{2}+\rho_{3}\right), \rho_{1}\left(\rho_{1}+\rho_{2}\right)\left(\rho_{1}+\rho_{2}+\rho_{3}\right) .
\end{gathered}
$$

Furthermore, in the same way as in the proof of Lemma 7.1, we can perform an equivariant connected sum of $M^{3} \sharp M^{3}$ with the 13 small covers listed in Theorem 6.6 along those 13 chosen fixed points such that the resulting 2-torus manifold is a small cover. In particular, this can still be carried out between $M^{3} \sharp M^{3}$ with some small covers chosen arbitrarily from the 13 small covers listed in Theorem 6.6. Now choose a representative of $\beta$ as a disjoint union of some small covers $M_{1}, \ldots, M_{t}$ of the 13 small covers listed in Theorem 6.6. Then one can perform an equivariant connected sum of $M^{3} \sharp M^{3}$ with $M_{1}, \ldots, M_{t}$ such that $M^{3} \sharp M^{3} \sharp M_{1} \sharp \cdots \sharp M_{t}$ is a small cover. Since $M^{3} \sharp M^{3}$ equivariantly bounds, by Corollary 2.2 one has that

$$
\beta=\left[M_{1}\right]+\cdots+\left[M_{t}\right]=\left[M_{1} \sqcup \cdots \sqcup M_{t}\right]=\left[M^{3} \sharp M^{3} \sharp M_{1} \sharp \cdots \sharp M_{t}\right] .
$$

Thus, $\beta$ contains a small cover as its representative.

\section{Acknowledgments}

I thank Professor M. Masuda for his valuable suggestions and comments, especially for conversation helpful to the argument of Proposition 2.3. I am also grateful to Professor R. E. Stong for his valuable suggestions and comments. In addition, I thank the referee for extremely careful reading of the original version and many valuable suggestions, comments and corrections.

\section{References}

[Allday and Puppe 1993] C. Allday and V. Puppe, Cohomological methods in transformation groups, Cambridge Studies in Advanced Mathematics 32, Cambridge University Press, Cambridge, 1993. MR 94g:55009 Zbl 0799.55001

[Alperin and Bell 1995] J. L. Alperin and R. B. Bell, Groups and representations, Graduate Texts in Mathematics 162, Springer, New York, 1995. MR 96m:20001 Zbl 0839.20001 
[Bao and Lü 2008] Z. Q. Bao and Z. Lü, "Manifolds associated with $\left(\mathbb{Z}_{2}\right)^{n}$-colored regular graphs", preprint, 2008. arXiv math.GT/0609557v4

[Bukhshtaber and Raŭ 1998] V. M. Bukhshtaber and N. Ră̆, "Toric manifolds and complex cobordisms", Uspekhi Mat. Nauk 53:2(320) (1998), 139-140. In Russian; translated in Russ. Math. Surv. 53:2 (1998), 371-373. MR 2000b:57047 Zbl 0967.57030

[Cai et al. 2007] M. Cai, X. Chen, and Z. Lü, "Small covers over prisms", Topology Appl. 154:11 (2007), 2228-2234. MR 2008d:57024

[Conner and Floyd 1964] P. E. Conner and E. E. Floyd, Differentiable periodic maps, Ergebnisse der Mathematik und ihrer Grenzgebiete (N.F.) 33, Springer, Berlin, 1964. MR 31 \#750 Zbl 0125.40103

[Davis and Januszkiewicz 1991] M. W. Davis and T. Januszkiewicz, "Convex polytopes, Coxeter orbifolds and torus actions", Duke Math. J. 62:2 (1991), 417-451. MR 92i:52012 Zbl 0733.52006

[Ewald 1996] G. Ewald, Combinatorial convexity and algebraic geometry, Graduate Texts in Mathematics 168, Springer, New York, 1996. MR 97i:52012 Zbl 0869.52001

[Grünbaum 2003] B. Grünbaum, Convex polytopes, 2nd ed., Graduate Texts in Mathematics 221, Springer, New York, 2003. MR 2004b:52001

[Guillemin and Zara 2001] V. Guillemin and C. Zara, "1-skeleta, Betti numbers, and equivariant cohomology”, Duke Math. J. 107:2 (2001), 283-349. MR 2002j:53110 Zbl 1020.57013

[Guillemin and Zara 2003] V. Guillemin and C. Zara, "The existence of generating families for the cohomology ring of a graph”, Adv. Math. 174:1 (2003), 115-153. MR 2004f:53099 Zbl 1020.57014 [Kosniowski and Stong 1979] C. Kosniowski and R. E. Stong, " $\left(\mathbb{Z}_{2}\right)^{k}$-actions and characteristic numbers”, Indiana Univ. Math. J. 28:5 (1979), 725-743. MR 81d:57027 Zbl 0437.57010

[Lü 2008] Z. Lü, "Graphs and $\left(\mathbb{Z}_{2}\right)^{k}$-actions", preprint, 2008. arXiv math.AT/0508643v6

[Stong 1970] R. E. Stong, "Equivariant bordism and $\left(\mathbb{Z}_{2}\right)^{k}$ actions", Duke Math. J. 37 (1970), 779785. MR $42 \# 6847$

Received September 7, 2008. Revised March 27, 2009.

\section{ZHI LÜ}

INSTITUTE OF MATHEMATICS

SCHOOL OF MATHEMATICAL SCIENCES

FUDAN UNIVERSITY

SHANGHAI 200433

CHINA

zlu@ fudan.edu.cn 